\title{
Warm gas in the rotating disk of the Red Rectangle: accurate models of molecular line emission ${ }^{\star}$
}

\author{
V. Bujarrabal ${ }^{1}$ and J. Alcolea ${ }^{2}$ \\ 1 Observatorio Astronómico Nacional (OAN-IGN), Apartado 112, 28803 Alcalá de Henares, Spain \\ e-mail: v.bujarrabal@oan.es \\ 2 Observatorio Astronómico Nacional (OAN-IGN), C/ Alfonso XII, 3, 28014 Madrid, Spain
}

Received 28 November 2012 / Accepted 27 February 2013

\begin{abstract}
Aims. We aim to study the excitation conditions of the molecular gas in the rotating disk of the Red Rectangle, the only postasymptotic-giant-branch object in which the existence of an equatorial rotating disk has been demonstrated. For this purpose, we developed a complex numerical code that accurately treats radiative transfer in $2 \mathrm{D}$, adapted to the study of molecular lines from rotating disks.

Methods. We present far-infrared Herschel/HIFI observations of the ${ }^{12} \mathrm{CO}$ and ${ }^{13} \mathrm{CO} J=6-5, J=10-9$, and $J=16-15$ transitions in the Red Rectangle. We also present our code in detail and discuss the accuracy of its predictions, from comparison with well-tested codes. Theoretical line profiles are compared with the empirical data to deduce the physical conditions in the disk by means of model fitting.

Results. We conclude that our code is very efficient and produces reliable results. The comparison of the theoretical predictions with our observations reveals that the temperature of the Red Rectangle disk is typically $\sim 100-150 \mathrm{~K}$, about twice as high as previously deduced from mm-wave observations of lower- $J$ lines. We discuss the relevance of these new temperature estimates for understanding the thermodynamics and dynamics of this prototype object, as well as for interpreting observations of other rarely studied post-AGB disks. Despite our sophisticated treatment of the line formation, our model cannot explain the relatively strong line-wing emission for intermediate- $J$ transitions. We argue that a model including a rotating disk only cannot reproduce these data and suggest that there is an additional extended (probably bipolar) structure expanding at about $7-15 \mathrm{~km} \mathrm{~s}^{-1}$.
\end{abstract}

Key words. stars: AGB and post-AGB - circumstellar matter - radio lines: stars - planetary nebulae: individual: Red Rectangle

\section{Introduction}

Disks orbiting post-asymptotic-giant-branch (post-AGB) stars are often postulated to explain the very energetic axial outflows that take place in this evolutionary phase (see e.g. Bujarrabal et al. 2001; Soker 2001; Balick \& Frank 2002; Frank \& Blackman 2004). Planetary and protoplanetary nebulae (PNe, $\mathrm{PPNe}$ ) very often show axisymmetric shapes and fast axial movements, which are thought to be caused by shock interaction between the very collimated post-AGB jets and the slow and isotropic AGB wind. According to simulations, mass accretion from disks rotating around post-AGB stars (with a magnetic field) can provide the energy and momentum required to explain the PPN dynamics via the ejection of very fast jets. The study of these rotating disks and their main physical conditions is therefore a basic requirement for understanding the post-AGB ejections, and therefore the spectacular and very fast evolution of the shape and dynamics of young PNe.

Disks/tori of molecular gas around post-AGB stars are commonly detected as the central part of protoplanetary and young planetary nebulae, although they are not observed to rotate, but to systematically expand (like the rest of the nebula).

\footnotetext{
* Appendices are available in electronic form at http://www . aanda.org
}

These expanding structures are usually thought to be mere remnants of the former AGB winds. See the cases of M 1-92 (Alcolea et al. 2007), M 2-9 (Castro-Carrizo et al. 2012), M 2-56 (Castro-Carrizo et al. 2002), etc. Yet there is one remarkable exception: the Red Rectangle.

The Red Rectangle is a well-known PPN that surrounds the A1 star HD 44179, a spectroscopic binary (e.g. Waelkens et al. 1996). In the visible and near-infrared (NIR), the reflection nebula is very extended (about $1^{\prime}$ ) and presents a conspicuous symmetry axis. It shows a spectacular X-shaped morphology, including a remarkable series of "ladder rungs" perpendicular to the symmetry axis (Cohen et al. 2004). Several models have been developed to explain these images, attempting to derive the dust distribution and illumination pattern (e.g. Men'shchikov et al. 2002; Koning et al. 2011) as well as the dynamics of the jet-shell interaction that probably shaped the nebula (Velázquez et al. 2011, and references therein).

The Red Rectangle is the only PPN in which a central disk has been actually observed to be in rotation (Bujarrabal et al. 2005), thanks to high-resolution maps of the CO $J=2-1$ and $J=1-0 \mathrm{~mm}$-wave lines. These maps show that the molecular gas in this source forms an equatorial rotating disk perpendicular to the optical nebula axis. Modeling of these observations indicates that the inner regions of the molecular disk, closer than $R_{\mathrm{kep}} \sim 8.4 \times 10^{15} \mathrm{~cm}$, are in Keplerian rotation around a central 
mass of about $1.7 M_{\odot}$ (for a distance of 710 pc; see Bujarrabal et al. 2005). In outer regions of the disk, farther than $R_{\mathrm{kep}}$ and up to the outer disk radius $R_{\text {out }} \sim 2.7 \times 10^{16} \mathrm{~cm}$, the gas shows a slow expansion of $\sim 0.8 \mathrm{~km} \mathrm{~s}^{-1}$ superimposed to rotation.

The orbiting material in the Red Rectangle was first proposed to explain several properties of this object, namely the probable presence of big grains in the nebula, the strong NIR excess, and the anomalous abundances found in the stellar atmosphere, but without any direct detection (see e.g. Waters et al. 1992; Waelkens et al. 1996; Jura et al. 1997). Following similar arguments, very compact rotating disks have also been proposed to surround other evolved stars, mainly in the post-AGB phase (see Jura \& Kahane 1999; Van Winckel 2003; De Ruyter et al. 2006; etc.), but again no direct detection of the velocity field has been obtained in them. In one of these objects, $89 \mathrm{Her}$, mm-wave maps show a very compact component (smaller than $\sim 5 \times 10^{15} \mathrm{~cm}$ ), whose low-velocity dispersion seems incompatible with expansion and supports the existence of an inner Keplerian disk (see Bujarrabal et al. 2007); this compact component is surrounded by an extended hour-glass-shaped halo in slow expansion.

A relatively high temperature was deduced in the Red Rectangle disk from the $J=2-1$ and $J=1-0$ maps, with $T \sim$ $65 \mathrm{~K}$ at $r=R_{\text {kep }}\left(8.4 \times 10^{15} \mathrm{~cm}\right.$, the distance at which the velocity regime changes) and typical values of $T \sim 100 \mathrm{~K}$ in the Keplerian inner region. Note however that low- $J$ CO transitions are poor probes of the kinetic temperature in such warm regions, because the $J=2-1$ line only requires about $15 \mathrm{~K}$ to be excited (the $J=1-0$ requiring $5.5 \mathrm{~K}$ ). Therefore, although those observations are very useful for studying the structure and dynamics of the warm molecule-rich regions, the derived temperatures are just a crude approximation, and so the excitation conditions in the Red Rectangle's rotating disk remained poorly probed, especially at the innermost regions. This problem also applies to the disk mass, though less critically, because errors in the temperature estimate in general may induce errors in the mass estimate. Very generally speaking, observations of transitions whose excitation energy is comparable to or somewhat higher than the excitation of a gas component, usually given by its kinetic temperature, are necessary to properly study that component.

Herschel/HIFI observations of high- $J$ molecular lines in the Red Rectangle have been recently published (Bujarrabal et al. 2012), including data of the ${ }^{12} \mathrm{CO}$ and ${ }^{13} \mathrm{CO} J=6-5$, $J=10-9$, and $J=16-15$ transitions (all of them, excepted the ${ }^{13} \mathrm{CO} J=16-15$ line, are clearly detected). The high spectral resolution of the heterodyne spectrometer HIFI yields accurate line profiles from which we can distinguish the contributions of different parts of the disk rotating at different velocities. The ${ }^{12} \mathrm{CO} J=6 / 10 / 16$ rotational levels are placed at $115 / 300 / 750 \mathrm{~K}$ above the ground level, and therefore can properly probe the excitation of the warm gas in the disk. Bujarrabal et al. (2012) concluded that the temperature deduced from the low- $J$ data was significantly underestimated, but using quite general considerations on the line emissivity as a function of the temperature and not a detailed modeling of the molecular excitation.

Transitions requiring still much higher excitation (over $3000 \mathrm{~K}$ ) have also been observed in the Red Rectangle using Herschel/PACS. Modeling of such data is particularly uncertain and beyond the scope of this paper. These lines must originate only from far inside and hot disk regions that are not probed at all by the mm-wave maps (and therefore are largely unknown), and the PACS observations do not provide information on the profile shape.
Accurate modeling is particularly important for a rotating disk, whose line excitation shows peculiar properties because it depends on photon trapping in a velocity regime that yields radiative interactions between very distant points and a quite complex structure of these coherence regions. Therefore, very complex calculations of radiative transfer and level population are necessary to really approach the line emission of such a structure. In this paper, we present an accurate treatment of radiative transfer in one and two dimensions, applied to the $\mathrm{CO}$ line emission from the Red Rectangle. The radiative transfer calculations for each of the transitions, coupled with the statistical equilibrium equations that give the populations of the relevant energy levels, are solved by the code simultaneously for all points of the molecule-rich cloud. Once the solution is attained after a complex iterative process, the resulting level populations are used to calculate the brightness distributions of a given line by solving the standard radiative transfer equation. The characteristics of the code are discussed in detail in Appendix A for both the $1 \mathrm{D}$ and $2 \mathrm{D}$ cases. We present extensive tests to the code, particularly in comparison with previous calculations and with results from the well-known large-velocity-gradient (LVG) approximation, and discuss the validity of different approximations to the line excitation under various conditions. We conclude that our code yields accurate calculations in the considered conditions, notably for a rotating disk.

We compare line profile predictions from our code with the Herschel/HIFI data of the Red Rectangle, which were carefully reanalyzed. The physical conditions in the Red Rectangle disk are deduced from model fitting, keeping the nebula model as similar as possible to that deduced by Bujarrabal et al. (2005); we confirm that the temperature was significantly underestimated from their analysis of low- $J$ observations.

\section{Nonlocal treatment of radiative transfer and line excitation}

We have developed a completely new code to treat radiative transfer and line excitation in one and two dimensions, adapted to interstellar clouds and circumstellar shells or disks. It is wellknown that molecular line formation from a gas cloud is basically given by the level populations in each point together with the cloud structure and kinetics. The level population in a given point is given by the rates of population from and depopulation to the other levels. We can assume statistical equilibrium for each level (its population becomes independent of time), because the typical rates are much faster than the typical evolution times of the physical conditions in the interstellar or circumstellar media.

The population transfer rates are mainly due to collisional and radiative transitions. Collisional rates, $C$, are assumed to be proportional to the local density (in our case, the gas is mostly composed of $\mathrm{H}_{2}$ ), since three-body encounters are very rare in our case, and are assumed to satisfy the microreversibility principle, which guarantees the Boltzmann distribution of populations for thermal equilibrium (with the thermal bath, i.e., when only collisions are relevant):

$C_{\mathrm{l}, \mathrm{u}}=C_{\mathrm{u}, 1} g_{\mathrm{u}} / g_{1} e^{-\frac{E_{\mathrm{u}}-E_{1}}{k T}}$,

where 1 and $u$ represent the lower and upper levels of the transition, $g$ and $E$ are the level statistical weights and energies, and $T$ is the gas kinetic temperature. Other symbols have their usual meanings. Collisional rates in $\mathrm{cm}^{3} \mathrm{~s}^{-1}$ units are usually obtained 
from ab initio quantum mechanical calculations of molecule encounters.

The transition rates of each radiative transition are given by the Einstein $A$ - and $B$-coefficients and the incident radiation at the resonant frequency. In this code version for $\mathrm{CO}$, we only include allowed rotational transitions in the vibrational $v=0$ state that satisfy $\Delta J= \pm 1$. The contribution of vibrational excitation to the emission of the $v=0$ rotational ladder is negligible for the high densities in the disk we are dealing with, see Sects. 2.2 and 4 , though it may be relevant in other cases. Of course, the treatment of the ro-vibrational lines is identical to that of the rotational ones, and the code can take them into account if necessary. The radiative transition probabilities are given by

$R_{\mathrm{u}, 1}=A_{\mathrm{u}, 1}+4 \pi B_{\mathrm{u}, 1} \bar{J}$

and

$R_{\mathrm{l}, \mathrm{u}}=4 \pi B_{\mathrm{l}, \mathrm{u}} \bar{J}$

$\bar{J}$ is the intensity $I(v)$ that arrives at the point integrated over all angles and frequencies (weighted with the normalized line profile $\Phi)$ :

$\bar{J}=\int J(v) \Phi(v) \mathrm{d} v, J(v)=\frac{1}{4 \pi} \int I(\theta, \phi) \mathrm{d} \Omega$.

The Einstein coefficients also satisfy the conditions necessary to yield a Boltzmann distribution when equilibrium with a radiation thermal bath takes place:

$B_{\mathrm{u}, 1}=\frac{c^{2}}{8 \pi h v^{3}} A_{\mathrm{u}, 1}, B_{1, \mathrm{u}}=\frac{c^{2}}{8 \pi h v^{3}} \frac{g_{\mathrm{u}}}{g_{1}} A_{\mathrm{u}, 1}$.

The relations between the $A$ and $B$ coefficients may vary from author to author, depending on the formulation taken for the rates $R$, but the dependence of $R$ on $A$ must remain unchanged.

The (local) line profile $\Phi$ is given by the local velocity dispersion and the corresponding Doppler shifts, since the natural line width is completely negligible in our case in which collisional and radiative transition rates do not exceed $\sim 1 \mathrm{~s}^{-1}$. We are implicitly assuming complete redistribution, i.e., that the line profile $\Phi$ is the same for absorption and emission; this approximation simplifies the treatment and is again fully satisfied in our case with very probable elastic collisions (line scattering is not very important in the low-probability $\mathrm{CO}$ transitions we are discussing).

The key problem is of course the calculation of $\bar{J}$ in each point of the cloud, which must be performed by solving the usual radiative transfer equation for resonant transitions, and therefore depends on the conditions of the other points. This introduces a very complex coupling between the excitation of the different parts of the cloud, and accordingly the solution of the whole problem is difficult in the general case. Several ways to solve it have been proposed, including approximations of the integrals by means of quadrature formulae, Monte Carlo methods, or approximate integration of the intensity obtained from ray tracing (see for instance Lucas 1976; Bernes 1979; van Zadelhoff et al. 2002). We adopt a ray tracing procedure, see Sect. 2.2.

Once $\bar{J}$ is calculated for all transitions, the code must solve the statistical equilibrium equations for the population $n_{i}$ of all levels $i$ in all points of the cloud:

$\dot{n}_{i}=\sum_{j}\left[n_{j} C_{j, i}+n_{j} R_{j, i}-n_{i} C_{i, j}-n_{i} R_{i, j}\right]=0$.
The solution is a set of level populations for all points consistent with the integrated intensity and transition rates. In practical cases, we must use the level populations to calculate again $\bar{J}$ for all transitions and points, and the system of equations results in an iterative process, where Eqs. (4) and (6) are alternatively solved.

Once convergence has been attained and the excitation of the treated molecule has been calculated, one can calculate the resulting line profiles by just solving the radiative transfer equation along the lines of sight for a number of frequencies around the resonant one, i.e., for a number of relative Doppler-equivalent velocities.

\subsection{The LVG or Sobolev approximation}

A simplified way to address the problem is possible when there is a significant velocity gradient in the molecular cloud. Under certain conditions (sufficiently high velocity and sufficiently high velocity gradient and, for instance, monotonically increasing or decreasing radial velocity), points separated by a significant distance do not interact radiatively because the relative Doppler shift is much higher than the local velocity dispersion. When the coherence region (where radiative interaction actually takes place) around a given point is small enough compared with the variation scale of the physical conditions, only the local properties are relevant for calculating the averaged intensity, which becomes

$\bar{J} \sim(1-\beta) S+\beta_{\mathrm{c}} I_{\mathrm{c}}$.

$\beta$ is the escape probability, which is a local function and depends on the velocity field. In the case of a radial field, $\beta$ depends on the local logarithmic gradient of the velocity $V(r)$ ( $r$ being the distance to the center): $\epsilon=\frac{\operatorname{dn}(V)}{\operatorname{dn}(r)}$. In the simplest case, for $\epsilon=1$, the (characteristic $L V G$ ) opacity $\tau$ is isotropic and

$\beta=\left(1-\mathrm{e}^{-\tau}\right) / \tau$.

$\beta$ is in fact the probability that a photon emitted from a point finally leaves the cloud in spite of (local) trapping; $\beta \sim 1$ in optically thin cases and $\beta \sim 1 / \tau$ in the optically thick limit. $\beta_{\mathrm{c}} I_{\mathrm{c}}$ represents the contribution of the radiation from continuum sources, which can often be written in two separated terms, the intensity of the continuum and $\beta_{\mathrm{c}}$, a generalized continuum escape probability that depends only on local properties.

This approximation was first developed by Sobolev (Sobolev 1960). A detailed and very understandable formalism can be found in Castor (1970), and some generalizations were discussed by Bujarrabal et al. (1980).

The LVG approximation enormously simplifies the problem, since the population of the energy levels at a given point only depends on local properties, though the system of Eq. (6) is strongly non-linear and an iterative numerical process is necessary (except for very low optical depths and obviously thermalized level systems). The LVG formalism is known to yield accurate solutions of the excitation state, even when the conditions are barely satisfied, see discussion on its validity in Appendix A. It contains most of the ingredients relevant in the line excitation, including trapping effects, yields very fast calculations, and, finally, does not require one to assume details of the cloud structure, dynamics, and physical conditions, of which we only know characteristic values in actual sources. In any case, the LVG codes can often be applied to estimate the molecular excitation (i.e., the level populations), but they are not accurate enough to estimate the resulting line intensity and profile, which must be 
calculated by solving the exact transfer equation across the cloud using the set of $n_{i}$ given by the LVG code. We compare LVG results with our non-local calculations in several cases and discuss the validity of this approximation in Appendix A.

\subsection{Our code}

As we show in Appendix A, for some velocity fields that are present in situations of astrophysical interest, the molecular excitation cannot be approached by means of simplified treatments, such as the LVG formalism. This is the case of rotating disks, in which radiative interaction between distant points takes place. We also show that some excitation effects, such as the underexcitation often found at the cloud edge, are similarly difficult to describe with these approximations. We have developed a new code to accurately solve the full radiative transfer equations in one and two dimensions, coupled with calculations of the level populations, whose general formalism has been described above. The leading idea of our code is that the geometrical parameters must be calculated as much as possible at the beginning and then used in all iterations.

The cloud is divided into a high number of small cells, within which we assume constant excitation conditions. The representative values of $\bar{J}$ for all transitions and of the level populations $n$ for all levels are calculated for the center of the cells. As mentioned, in our code $\bar{J}$ is calculated following the ray-tracing procedure. A sufficiently high number of ray directions are considered that sample the whole space of the directions. We calculate the paths of the model rays that traverse other cells (i.e., the lengths traversed by the ray in each cell, the relative velocity between the cells in the ray direction, etc.). The rays are defined by means of their azimuth and elevation angles with respect to the symmetry axis of the model nebula (our 2D calculations assume axial symmetry). This procedure is not optimized for 1D calculations in a spherical cloud, but the method was kept since our goal is in fact its extension to 2D calculations. For an initial set of level populations, we calculate the intensity $I$ for all rays arriving at each point and for a sufficiently high number of frequencies within the local line profile, solving the radiative transfer equation discretized in the high number of cells traversed by the rays. We then calculate $\bar{J}$ for the considered point, approaching the integral in Eq. (4) by the addition (with the corresponding weights) of the intensities arriving along the rays for all the considered directions and frequencies. With the set of values of $\bar{J}$ for all radiative transitions, we calculate the level populations $\left\{n_{i}\right\}$ (from Eq. (6)) and, using them, a new iteration is performed. The efficiency of the iterative process basically depends on the total opacity; typically, the number of iterations is of the same order as the highest opacity in the cloud, since the information is transferred from one point to another by steps of about $1 / \tau$ of the total size in each iteration. The velocity field is also important in the computing time. When the macroscopic velocity is very high, many velocity points must be considered and, in general, the coherence region (as defined in Sect. 2.1) becomes very small and a very fine cell net is required; in this limit, an LVG-like approach is obviously preferable. We show details of the computer code in Appendix A, where we also extensively compare the results of our code with previous calculations and with LVG calculations, with very satisfactory results.

Several conclusions can be reached from our detailed analysis in Appendix A:

1. Our code is fully consistent with previously published calculations and with results obtained from the LVG approximation, when it can be applied. We conclude that the results from the code are reliable.

2. Accurate non-local calculations are necessary in the case of rotating disks, in which the $\mathrm{CO}$ line formation cannot be adequately approximated with LVG or LVG-like codes.

3. Nevertheless, LVG estimates of the level populations (not of the line profile) are reasonably accurate in many other cases, even when the high-velocity gradient requirement is barely satisfied, and can be used in a wide variety of situations. Another conclusion can be reached from direct inspection of the radiative transition probabilities and is confirmed by our calculations:

4. The CO low- $J$ transitions are easily thermalized. Their low Einstein A-coefficients, $\sim 10^{-7} \mathrm{~s}^{-1}$, guarantee thermalization for densities $n \gtrsim 10^{4} \mathrm{~cm}^{-3}$ (e.g. Bujarrabal et al. 1997). Accordingly, our calculations confirm that the previous analysis of the mm-wave maps by Bujarrabal et al. (2005; where LTE was assumed) are basically correct. However, the Einstein coefficient increases very fast with $J$, approximately as $J^{3}$; for instance, $A(J=16-15)=4 \times 10^{-4} \mathrm{~s}^{-1}$, and this line is thermalized only for $n \gtrsim 5 \times 10^{7} \mathrm{~cm}^{-3}$. Typical densities in the object we study here, the Red Rectangle disk, are $n\left(R_{\mathrm{kep}}\right) \sim 4 \times 10^{5} \mathrm{~cm}^{-3}$ (Bujarrabal et al. 2005). Therefore, in our case (and in many others), thermalization cannot be assumed for the high- $J$ lines and complex calculations are necessary.

\section{Herschel/HIFI and IRAM $30 \mathrm{~m}$ data of the Red Rectangle}

We present data of mm- and submm-wave lines of ${ }^{12} \mathrm{CO}$ and ${ }^{13} \mathrm{CO}$ in the Red Rectangle, which are compared with model predictions. The data have been taken with the IRAM $30 \mathrm{~m}$ telescope and the HIFI instrument onboard the Herschel Space Observatory (HSO). The spectra are shown in Fig. 1 (also Fig. 3 and Figs. B1-B3).

Data for the $J=2-1$ line were taken with the EMIR receivers of the IRAM $30 \mathrm{~m}$ telescope. These data are new and belong to a larger survey of molecular gas disks around postAGB objects, which will be published in a forthcoming paper. The mm-wave data were calibrated in the standard way at the $30 \mathrm{~m}$ instrument in units of main-beam Rayleigh-Jeansequivalent temperature $T_{\mathrm{mb}}$. These values were re-scaled after the observation of strong $\mathrm{CO}$ lines of well-known emitters (CW Leo, CRL 618, and NGC 7027). After these corrections, we estimate the absolute incertitude in fluxes to be approximately $\pm 20 \%$. More details on the observation will be given in the paper dedicated to the disk survey.

Data for the $J=6-5$ lines and above were obtained with the wide band spectrometers of the Herschel/HIFI instrument. These data were already discussed by Bujarrabal et al. (2012), where all details on the observations and data reduction can be found. Here we present data obtained after an improved reduction, which include the most recent values for the calibration parameters of the instrument (Roelfsema et al. 2012). Intensities are also given in units of $T_{\mathrm{mb}}$. For the HIFI data, uncertainties in the calibration are larger and mostly due to uncertainties in the side-band gain calibration and the baseline, with values of about $20 \%$ for the $J=6-5$ and $J=10-9$ lines and of $30 \%$ for ${ }^{12} \mathrm{CO} J=16-15$.

A summary of the main line parameters (including the observed line peak, area, and uncertainties) is given in Table 1, together with some parameters of the model fitting. 


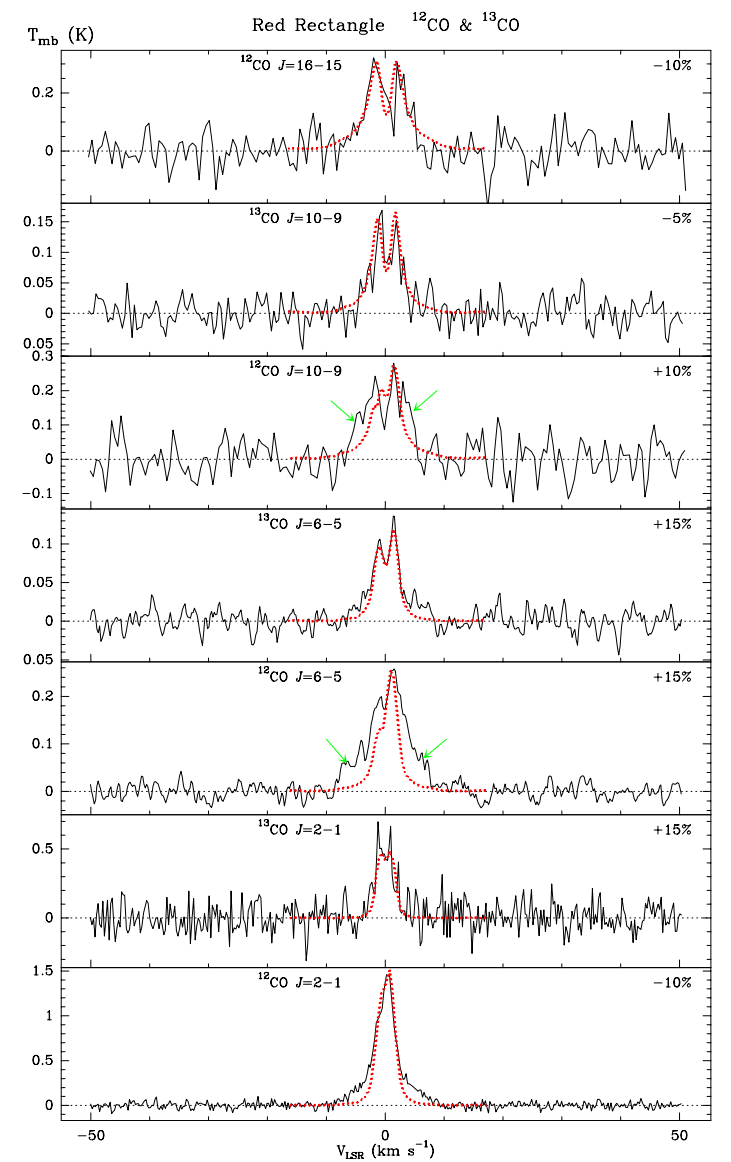

Fig. 1. Observed profiles of the $\mathrm{mm}$ - and submm-wave transitions (black), Sect. 2. We also show (red points) our best-fit model predictions from a rotating disk, which adequately reproduces the line cores, but underestimates the line-wing emission of some lines (green arrows), see Sect. 4. The values of the free scale parameter in the model predictions that account for uncertainties in the calibration are indicated in the upper-right corners.

\section{Physical conditions in the molecular disk of the Red Rectangle}

We have applied the most complex $2 \mathrm{D}$ version of our code to fit our observations of the ${ }^{12} \mathrm{CO}$ and ${ }^{13} \mathrm{CO}$ lines. A sophisticated treatment is necessary because, as we have seen in Sect. 2.2, simplified treatments (LVG or LTE) are inaccurate. In our numerical calculations, the code typically considered more than 20 rotational levels, about 800 cells, and about 200 rays (arriving at each cell); we verified that convergence is attained for these values (as for the rest of the numerical parameters entering the calculations). The contribution of vibrational excitation to the emission of the $v=0$ rotational ladder is negligible in our case (as in most evolved nebulae), even in the presence of continuum IR sources. A general discussion on the effects of the vibrational excitation for PPNe and PNe will be presented in Santander-García et al. (in prep.), see a preliminary report in Santander-García et al. (2012). The excitation calculated from the code was used to calculate the brightness of the observed transitions for a high number of impact parameters in the direction of the line of sight and of LSR velocities. These distributions were convolved with Gaussian beams to obtain predicted line profiles directly comparable with the observations. Simulations of the interferometric observations of ${ }^{12} \mathrm{CO} J=1-0$ and $J=2-1$ by Bujarrabal et al. (2005) were also performed by convolution with the synthesized beams obtained in this work (using an additional code similar to the one described in it); we verified that for our new models the predicted low- $J$ maps are compatible with these observations within the uncertainties. In the calculations presented here we used the most recent collisional rates from the LAMBDA database $^{1}$, (Schöier et al. 2005; rates calculated by Yang et al. 2010) for collisions with ortho- and para- $\mathrm{H}_{2}$, for which we assumed an abundance ratio of 3. (Results are very slightly dependent on the assumed ratio and we cannot be sure under which excitation conditions $\mathrm{H}_{2}$ was formed.) Collisions with other gas components, notably He, are not considered, so the derived density represents the total density and not only the density of $\mathrm{H}_{2}$.

As mentioned (Sect. 1; Bujarrabal et al. 2012), the emission from the high- $J$ transitions in the Red Rectangle seems to be too intense to be explained by the relatively low temperature deduced from our analysis of low- $J$ lines (Bujarrabal et al. 2005), which are not suitable to estimate the temperature in warm gas (Sect. 1). However, the model by Bujarrabal et al. (2005) explained their high-resolution maps very well, therefore we tried to keep the disk structure and dynamics deduced in that paper as far as possible. Only the disk width was not clearly determined from these data, because the deduced value was smaller than the telescope resolution in the direction of the axis; the (slight) inclination of the disk plane with respect to the line of sight also hampers this estimation.

Calculations with our new code made with exactly the same nebula model as deduced by Bujarrabal et al. (2005) confirm that too low emission in the Herschel lines is then predicted, in particular the intensity of the $J=16-15$ transition of ${ }^{12} \mathrm{CO}$ would be about $1 / 3$ of the observed intensity. It is therefore necessary to increase the temperature of the central regions from which the high- $J$ transitions originate. The line intensities are well reproduced if we increase the temperature by about a factor two, to $T\left(R_{\mathrm{kep}}\right) \sim 100-150 \mathrm{~K}$ (we recall that $R_{\text {kep }}$ is a characteristic point in the disk at which the dynamical regime changes from purely Keplerian to rotation plus a slow expansion; see more details on the model and the parameter definition in Bujarrabal et al. 2005). The physical conditions finally deduced for the molecular disk in the Red Rectangle are shown in Table 2, in which we indicate in bold-face characters the values that are significantly different from those of the original nebula model by Bujarrabal et al. (2005). Indeed, only the gas temperature field has changed significantly compared to that model. We had only to assume one new parameter, the ${ }^{12} \mathrm{CO} /{ }^{13} \mathrm{CO}$ abundance ratio, which was assumed to be equal to 10. We compare in Fig. 1 the observed profiles and the predictions using these parameters. To account for observational calibration incertitudes (Sect. 3), we introduced an intensity scale tolerance in our fitting; the adopted scale factors are given in Table 1 (together with the expected calibration uncertainties) and Fig. 1 (in the upper-right corners of each panel). The distributions of the velocity, density, and temperature in the best-fit model nebula are shown in Fig. 2.

Other mechanisms to increase the relative intensity of the $J=16-15$ line are found not to be efficient enough to reproduce the observed intensity values. For instance, high line opacities tend to increase the population of the high- $J$ levels by means of photon trapping. The ${ }^{12} \mathrm{CO} J=16-15$ line is only moderately opaque in our case, but the opacity of the intermediate- $J$ lines is high. This effect indirectly leads to an increase of the $J=16$ and 15 level populations. Our calculations, which accurately include the overexcitation effect due to trapping, show that, under the expected conditions, this effect is not enough

1 http://www.strw.leidenuniv.nl/ moldata 
Table 1. CO lines: summary of observational parameters and fitting results.

\begin{tabular}{|c|c|c|c|c|c|c|c|c|c|c|c|}
\hline \multirow[t]{2}{*}{ Species } & \multirow[t]{2}{*}{ Line } & \multirow{2}{*}{$\begin{array}{c}\text { Rest freq. } \\
(\mathrm{GHz})\end{array}$} & \multirow{2}{*}{$\begin{array}{c}\text { Peak } \\
(\mathrm{K})\end{array}$} & \multirow{2}{*}{$\begin{array}{c}\text { Area }^{\dagger} \\
\left(\mathrm{K} \mathrm{km} \mathrm{s}^{-1}\right)\end{array}$} & \multirow{2}{*}{$\begin{array}{c}\mathrm{rms}^{\ddagger} \\
(\mathrm{K})\end{array}$} & \multirow{2}{*}{$\begin{array}{c}\text { Resol. } \\
\left(\mathrm{km} \mathrm{s}^{-1}\right)\end{array}$} & \multirow{2}{*}{$\begin{array}{l}\text { HPWB } \\
\left({ }^{\prime \prime}\right)\end{array}$} & \multirow[t]{2}{*}{$T_{\mathrm{mb}} / T_{\mathrm{a}}^{*}$} & \multirow{2}{*}{$\begin{array}{l}\text { Calib. } \\
\text { uncer. }\end{array}$} & \multicolumn{2}{|c|}{ Model } \\
\hline & & & & & & & & & & scale factor & peak $(\mathrm{K})$ \\
\hline${ }^{12} \mathrm{CO}$ & $J=16-15$ & 1841.346 & 0.31 & 2.05 & 0.055 & 0.57 & $11^{\prime \prime} 6$ & 1.4 & $30 \%$ & 0.90 & 0.31 \\
\hline${ }^{13} \mathrm{CO}$ & $J=10-9$ & 1101.350 & 0.17 & 0.81 & 0.02 & 0.41 & $19 ! ' 1$ & 1.3 & $30 \%$ & 0.95 & 0.17 \\
\hline${ }^{12} \mathrm{CO}$ & $J=10-9$ & 1151.985 & 0.27 & 1.77 & 0.05 & 0.52 & $18^{\prime \prime} 3$ & 1.5 & $15 \%$ & 1.10 & 0.27 \\
\hline${ }^{13} \mathrm{CO}$ & $J=6-5$ & 661.067 & 0.14 & 0.66 & 0.012 & 0.23 & 32.0 & 1.3 & $15 \%$ & 1.15 & 0.13 \\
\hline${ }^{12} \mathrm{CO}$ & $J=6-5$ & 691.473 & 0.26 & 1.92 & 0.015 & 0.21 & $30 .^{\prime} 8$ & 1.3 & $15 \%$ & 1.15 & 0.26 \\
\hline${ }^{13} \mathrm{CO}$ & $J=2-1$ & 220.399 & 0.65 & 2.33 & 0.09 & 0.27 & $12^{\prime \prime} 5$ & 1.7 & $20 \%$ & 1.15 & 0.5 \\
\hline${ }^{12} \mathrm{CO}$ & $J=2-1$ & 230.538 & 1.47 & 7.07 & 0.035 & 0.25 & $12^{\prime \prime} 0$ & 1.7 & $20 \%$ & 0.90 & 1.5 \\
\hline
\end{tabular}

Notes. ${ }^{(\dagger)}$ In the interval $[-10:+10] \mathrm{km} \mathrm{s}^{-1} \cdot{ }^{(\grave{\ddagger})}$ For the given resolution.

Table 2. Structure and physical conditions in the molecular disk in the Red Rectangle derived from our model fitting of the CO data.

\begin{tabular}{|c|c|c|c|c|}
\hline \multirow[b]{2}{*}{ Parameter } & \multicolumn{2}{|r|}{ Inner disk $\left(r<R_{\mathrm{kep}}\right)$} & \multicolumn{2}{|c|}{ Outer disk $\left(r>R_{\mathrm{kep}}\right)$} \\
\hline & Law & Values & Law & Values \\
\hline Outer radius & \multicolumn{2}{|r|}{$R_{\mathrm{kep}}=8.4 \times 10^{15}\left(\frac{D(\mathrm{pc})}{710}\right) \mathrm{cm}$} & \multicolumn{2}{|r|}{$R_{\text {out }}=2.7 \times 10^{16}\left(\frac{D(\mathrm{pc})}{710}\right) \mathrm{cm}$} \\
\hline Disk & \multirow[t]{2}{*}{ linear } & $H\left(R_{\mathrm{kep}}\right)=1.3 \times 10^{16}\left(\frac{D(\mathrm{pc})}{710}\right) \mathrm{cm}$ & \multirow[t]{2}{*}{ constant } & \multirow[t]{2}{*}{$H=1.3 \times 10^{16}\left(\frac{D(\mathrm{pc})}{710}\right) \mathrm{cm}$} \\
\hline thickness & & $H(0)=6.5 \times 10^{15}\left(\frac{D(\mathrm{pc})}{710}\right) \mathrm{cm}$ & & \\
\hline $\begin{array}{l}\text { Tangential } \\
\text { velocity }\end{array}$ & $\begin{array}{l}V_{\mathrm{t}} \propto 1 / \sqrt{r} \\
(\text { Keplerian })\end{array}$ & $\begin{array}{c}V_{\mathrm{t}}\left(R_{\mathrm{kep}}\right)=1.65 \mathrm{~km} \mathrm{~s}^{-1} \\
\left(\text { central mass: } 1.7\left(\frac{D(\mathrm{pc})}{710}\right) M_{\odot}\right)\end{array}$ & $\begin{array}{c}V_{\mathrm{t}} \propto 1 / r \\
\text { (ang. mom. cons.) }\end{array}$ & $V_{\mathrm{t}}\left(R_{\mathrm{kep}}\right)=1.65 \mathrm{~km} \mathrm{~s}^{-1}$ \\
\hline $\begin{array}{l}\text { Expansion } \\
\text { velocity }\end{array}$ & & $0 \mathrm{~km} \mathrm{~s}^{-1}$ & $V_{\exp } \propto \sqrt{a+b / r}$ & $\begin{array}{c}V_{\exp }\left(R_{\text {kep }}\right)=1.6 \mathrm{~km} \mathrm{~s}^{-1} \\
V_{\exp }\left(R_{\text {out }}\right)=0 \mathrm{~km} \mathrm{~s}^{-1}\end{array}$ \\
\hline $\begin{array}{l}\text { Turbulence } \\
\text { velocity }\end{array}$ & constant & $\sigma_{\text {turb }}=0.2 \mathrm{~km} \mathrm{~s}^{-1}$ & constant & $\sigma_{\text {turb }}=0.2 \mathrm{~km} \mathrm{~s}^{-1}$ \\
\hline Temperature & $\begin{array}{l}T \propto 1 / r^{\alpha_{T}} \\
n \propto 1 / r^{\alpha_{n}}\end{array}$ & $\begin{array}{c}T\left(R_{\text {kep }}\right)=\mathbf{9 5} \mathrm{K} \\
\alpha_{\mathrm{T}}=\mathbf{1} \\
n\left(R_{\mathrm{kep}}\right)=4 \times 10^{5}\left(\frac{710}{D(\mathrm{pc})}\right) \mathrm{cm}^{-3} \\
\alpha_{n}=2.2\end{array}$ & $T \propto 1 / r^{\alpha_{T}}$ & $\begin{array}{c}T\left(R_{\mathrm{kep}}\right)=150 \mathrm{~K} \\
\alpha_{\mathrm{T}}=0.7 \\
n\left(R_{\mathrm{kep}}\right)=110^{5}\left(\frac{710}{D(\mathrm{pc})}\right) \mathrm{cm}^{-3} \\
\alpha_{n}=2.2\end{array}$ \\
\hline \multicolumn{2}{|c|}{ Other parameters } & Law & Values & Comments \\
\hline \multicolumn{3}{|c|}{$\begin{array}{l}\text { Axis inclination from the plane of the sky } \\
\text { Axis inclination in the plane of the sky (PA) }\end{array}$} & $\begin{array}{c}5^{\circ} \\
11^{\circ}\end{array}$ & $\begin{array}{l}\text { from optical and CO data } \\
\text { from optical and CO data }\end{array}$ \\
\hline \multicolumn{3}{|c|}{ Distance } & 380 and $710 \mathrm{pc}$ & various arguments (Sect. 4) \\
\hline \multicolumn{2}{|c|}{${ }^{12} \mathrm{CO}$ relative abundance } & constant & $2 \times 10^{-4}$ & from $\mathrm{CO}$ data \\
\hline \multicolumn{2}{|c|}{${ }^{13} \mathrm{CO}$ relative abundance } & constant & $2 \times 10^{-5}$ & from $\mathrm{CO}$ data \\
\hline \multicolumn{2}{|c|}{ LSR systemic velocity } & & $-0.2 \mathrm{~km} \mathrm{~s}^{-1}$ & from CO data \\
\hline
\end{tabular}

Notes. Dependence on the assumed distance to the source is given in the relevant cases, but we favor a distance of $710 \mathrm{pc}$. We show in bold-face characters the values that change significantly compared to those deduced from the low- $J$ lines only; in practice this is only the gas temperature.

by itself to explain the observed intensities. On the other hand, we cannot increase the line opacity (for instance by assuming a higher $\mathrm{CO}$ abundance) without affecting the intensities of the other lines, and the calculations then contradict the observational data.

The model fitting, particularly in the line core, is satisfactory, see Fig. 1; the differences between calculations and observations are clearly smaller that the observational uncertainties. Because of the many parameters involved in the very complex disk model (not all of which are allowed to vary with the same degree of freedom) and the various sources of uncertainty in the data (sometimes not described by a normal distribution, such as the calibration uncertainty and the line wings, which are not explained by our models), we did not try to find the best fitting in a more systematic way, e.g. from a chi-square approach. There is, however, an obvious excess in the observed profiles at LSR velocities $\sim \pm 5-7 \mathrm{~km} \mathrm{~s}^{-1}$ (see arrows in Fig. 1). We were unable to reproduce this line-wing excess, even allowing variations of other disk parameters, see discussion in Sect. 4.1.

In Appendix B we estimate the uncertainty of our temperature determination, which is found to be about $20 \%$ from a comparison of predictions and observations for which we kept the other parameters constant. We also show that these uncertainty values are not significantly higher when we also allow moderate changes in the model structure and in the gas density. We recall that, in any case, the temperature distribution and structure of the very inner region cannot be well determined, because we severely lack spatial information on them: the mm-wave maps by Bujarrabal et al. (2005) attained an angular resolution equivalent to about $10^{16} \mathrm{~cm}$ and were not very sensitive to variations in the temperature, and, on the other hand, our observations of FIR lines are useful for measuring the temperature but have a still 


\section{Bujarrabal and J. Alcolea: The Red Rectangle rotating disk}

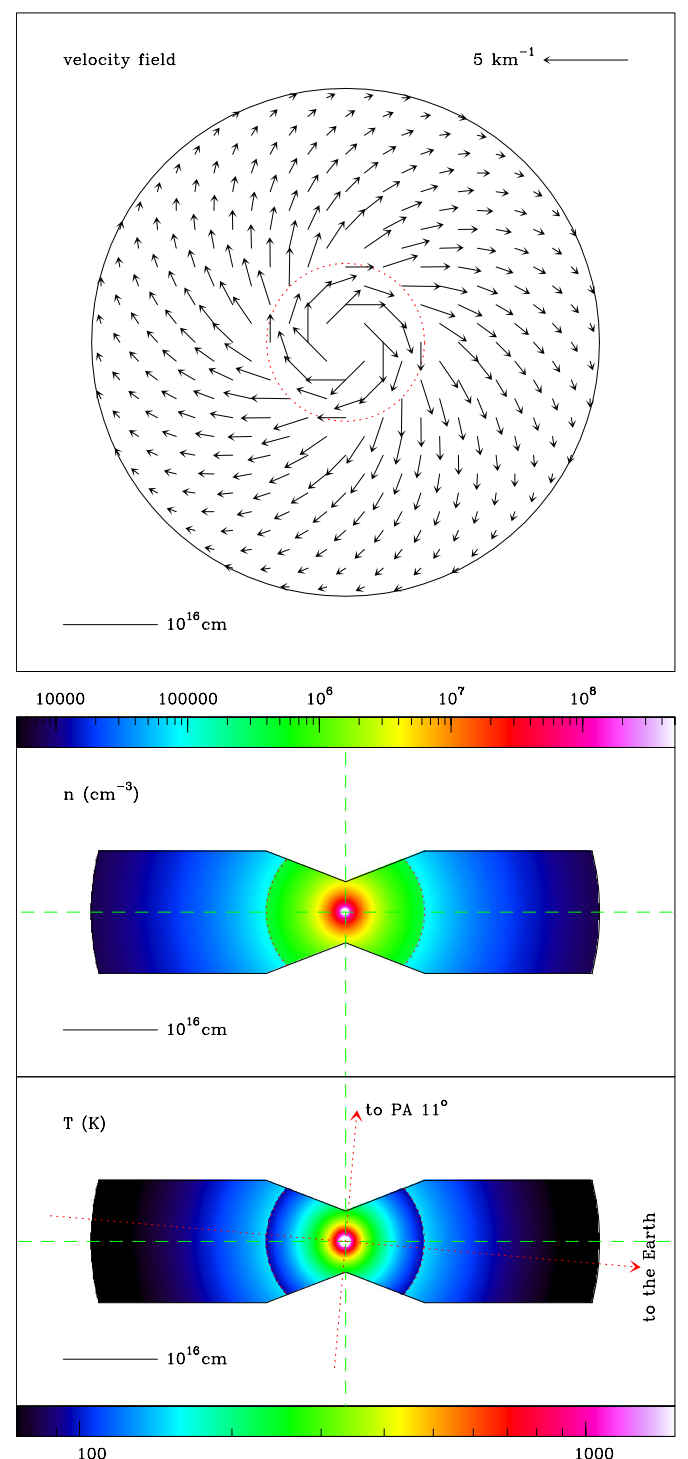

Fig. 2. Velocity and physical conditions in the best-fit model disk. We represent the projection of the gas velocity on the equatorial plane (upper panel, disk seen face-on) and the density and temperature fields across the disk (seen edge-on). The red circle indicates the value of $R_{\mathrm{kep}}$, from which the dynamical regime changes (see text).

poorer resolution (though assuming Keplerian rotation introduces a very useful but indirect relation between size and velocity). We hope that future high- $J$, high-resolution mapping will enable us to study the very inner regions of the Red Rectangle disk better.

\subsection{Properties of the gas responsible for the line-wing emission excess}

As we have seen, it is possible to reproduce most of the features identified in our profiles using a rotating disk model very similar to that deduced from the low- $J$ observations, but with significantly higher temperatures. However, our calculations indicate that the relatively wide line-wings observed in $J=6-5$ (and less clearly in other transitions, see green arrows in Fig. 1) are not explained by models of this kind, even if we allow variations of parameters other than the gas temperature or include new components. For instance, we compare in Fig. 3 the observations and the model predictions using the same physical conditions as for

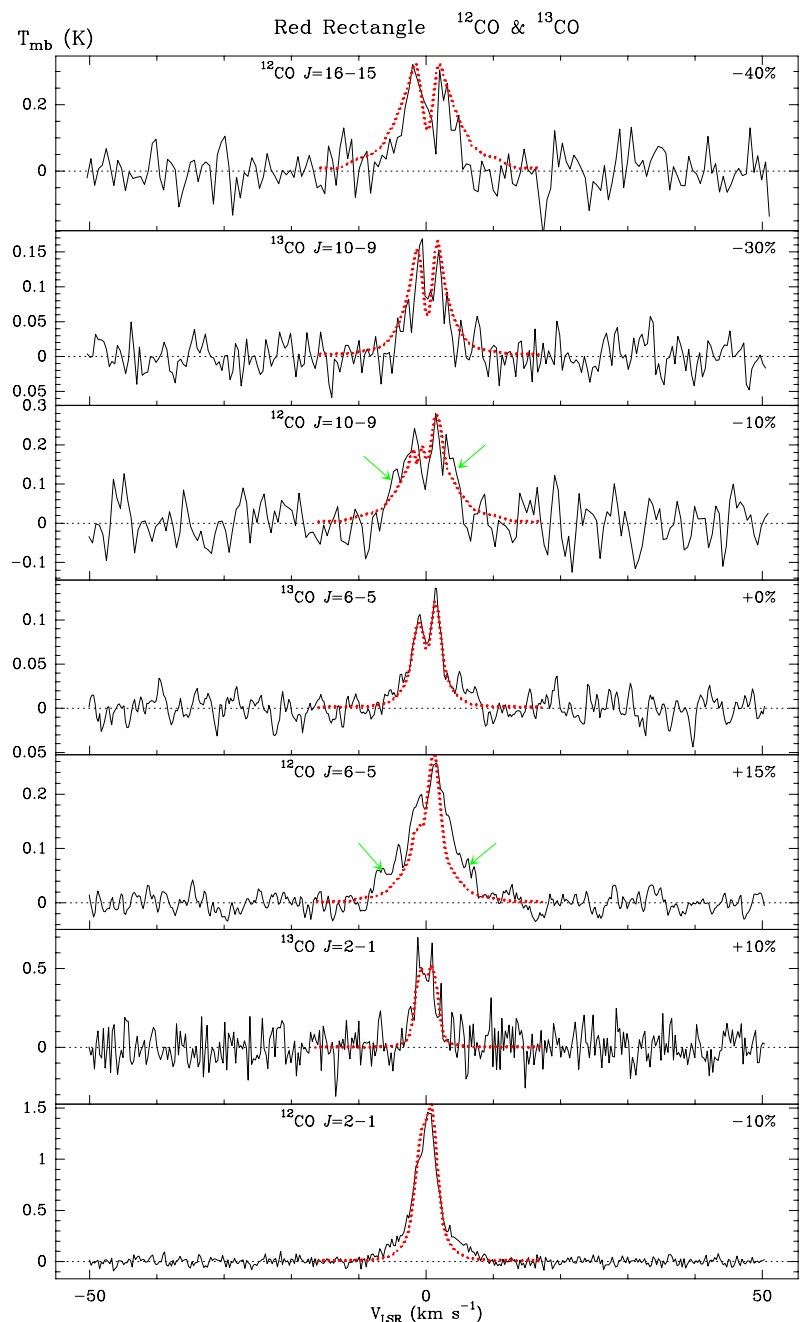

Fig. 3. Observed profiles of the $\mathrm{mm}$ - and submm-wave transitions (black) and predictions of our code (red points), assuming that the disk region closer than $2 \times 10^{15} \mathrm{~cm}$ to the axis is hotter and denser by a factor 2 than in our standard model, Table 2 . The symbols have the same meaning as in Fig. 1, note the values of the free scale parameter used in this case.

Fig. 1, but assuming an increase by a factor 2 in the temperature and density in the disk region closer than $2 \times 10^{15} \mathrm{~cm}$ to the axis. Evidently, the discrepancy in the wings of the intermediateexcitation lines is somewhat lower but it persists, and the intensity of the $J=16-15$ line, mostly in its wings, is now too high. Increasing the excitation of the inner disk regions even more yields still much stronger wings in the $J=16-15$ line.

We think that a model that only includes a rotating disk of this kind cannot explain the observed line-wing excess. A simple reasoning can help to understand the reasons for this:

We have detected emission at a level of about $T_{\mathrm{mb}}(J=6-5)$ $\sim 50 \mathrm{mK}$ from gas rotating at velocities of up to about $7.5 \mathrm{~km} \mathrm{~s}^{-1}$. If the dynamics is the Keplerian one used here (or similar), such a velocity only appears at a short distance from the star, $r \sim 4 \times 10^{14} \mathrm{~cm}$, which means an emitting region size of about 0 '07. Taking into account the strong beam dilution at this frequency, the brightness temperature in this region should be very high, $T_{\mathrm{B}} \sim 9000 \mathrm{~K}$. Except for very peculiar situations, the strongest line emission from molecular gas appears when the level populations are thermalized and the line is optically thick, then the brightness is equal to the kinetic temperature. Therefore, 


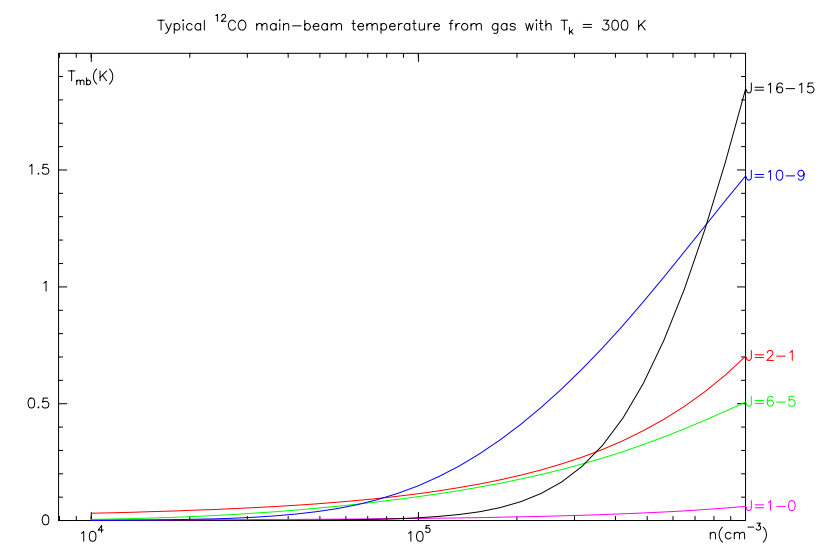

Fig. 4. Typical main-beam brightness temperature as a function of the density expected from the expanding gas component proposed to be responsible for the line-wing emission in intermediate-excitation $\mathrm{CO}$ lines (Sect. 4.1).

the kinetic temperature in this region must satisfy $T \gtrsim 10000 \mathrm{~K}$ to be able to account for the observed intensity.

Such high temperatures are surprising for gas placed at 26 AU from this A1-type star and one would not expect a high $\mathrm{CO}$ abundance in such a hot gas, accordingly, this $\mathrm{CO}$ excitation state must be considered to be at the limit of the possible situations. But even if these conditions are really present, they would produce a too strong emission in the $J=16-15$ transition. The high opacity we must assume in the $J=6-5$ transition (from this small region) requires densities higher than $10^{8} \mathrm{~cm}^{-3}$ (in agreement with the density laws we are dealing with), for which all transitions up to $J=16-15$ must also be thermalized and a fortiori be opaque, showing a brightness temperature similar to that of $J=6-5$. Taking into account the relatively low beam dilution for the $J=16-15$ observations (about seven times lower than for $J=6-5)$, we expect $T_{\mathrm{mb}}(J=16-15) \sim 350 \mathrm{mK}$ for LSR velocities $\sim \pm 7 \mathrm{~km} \mathrm{~s}^{-1}$. This evidently contradicts the observational data, which show values lower than about $50 \mathrm{mK}$ at these velocities and a line peak of $\sim 300 \mathrm{mK}$.

Therefore, the only way to explain this wide emission in $J=6-5$ is to assume that it comes from a more extended region, in which the relatively high velocity cannot be dominated by rotation. The excitation conditions can then be relaxed. We performed LVG calculations for a very preliminary and simplified case. We assumed gas at a typical distance from the center of $10^{16} \mathrm{~cm}$ (therefore occupying an angular region of about $3 \operatorname{arcsec}^{2}$ ), expanding at $15 \mathrm{~km} \mathrm{~s}^{-1}$ and with a temperature of $300 \mathrm{~K}$; the density was assumed to be a variable parameter. Results are shown in Fig. 4. As expected, the emission of this expanding gas component would be undetectable in ${ }^{12} \mathrm{CO} J=16-15$ for moderate densities, not much higher than $10^{5} \mathrm{~cm}^{-3}$, at which $J=2-1, J=6-5$, and $J=10-9$ show an intensity of about $0.1 \mathrm{~K}$. We took a typical velocity of about $15 \mathrm{~km} \mathrm{~s}^{-1}$, assuming that the observed LSR velocities result from a high angle between the line of sight and the velocity direction, but very similar results are obtained, for instance, taking an expansion velocity of about $7 \mathrm{~km} \mathrm{~s}^{-1}$. The calculated brightness values are very similar to the brightness excess shown by the observed lines at $\pm 4-7 \mathrm{~km} \mathrm{~s}^{-1}$ LSR and can then explain the observed features. A still better agreement can be found if we assume that the excess of the $J=10-9$ line at $\pm 4 \mathrm{~km} \mathrm{~s}^{-1}$ comes from a slightly denser region, while more diffuse gas would also contribute to the excess in $J=6-5$ at $\pm 6 \mathrm{~km} \mathrm{~s}^{-1}$; but a deeper discussion of this very uncertain component is meaningless and will only show these examples of the possible properties of the emitting gas.

In a similar object, $89 \mathrm{Her}$, molecule-rich gas in expansion that forms an hourglass-like structure has been mapped (Sect. 1, Bujarrabal et al. 2007). In the Red Rectangle, the wide optical nebula is known to be in expansion, although no $\mathrm{CO}$ emission was detected from it in the interferometric observations by Bujarrabal et al. (2005), perhaps because of the low expected brightness ( $\lesssim 5 \mathrm{~K}$ for the example presented above, which is lower than the continuum level) and its relatively wide extent. A moderate increase in the sensitivity should lead to the detection of this component.

In summary, we suggest that the emission excess found in the wings of some observed lines, particularly obvious in our $J=6-5$ profile, is due to the existence of a moderately extended component of molecule-rich gas in expansion. No counterpart would be expected in very high- $J$ transitions. We do not attempt to describe this component in detail, because we currently know too little about its properties.

\section{Conclusions}

We presented a new sophisticated code for calculating molecular line excitation in two dimensions. The extensive tests presented in Appendix A lead us to conclude that the numerical results are accurate. The code in particular yields various line profiles (after convolving the brightness distributions with the observational angular responses) that can be directly compared with observational results.

We have used our code to study the physical conditions in the equatorial disk of the Red Rectangle. This well-known protoplanetary nebula is the only AGB or post-AGB nebula in which a disk in rotation has been properly identified, thanks to $\mathrm{mm}$ wave maps of ${ }^{12} \mathrm{CO} J=2-1$ and $J=1-0$ by Bujarrabal et al. (2005). These authors modeled the disk to reproduce the images and derived a reliable description of its structure and dynamics. But such low- $J$ transitions, which require a very low temperature to be excited, are not useful for studying the excitation state of the gas when its temperature is $\gtrsim 50 \mathrm{~K}$, which is the case of most of the molecular gas in the Red Rectangle.

Herschel/HIFI observations of ${ }^{12} \mathrm{CO}$ and ${ }^{13} \mathrm{CO} J=6-5$, $J=10-9$, and $J=16-15$ recently provided high $\mathrm{S} / \mathrm{N}$ profiles of these transitions, see Bujarrabal et al. (2012). These authors concluded from a preliminary analysis of the excitation conditions that the kinetic temperatures derived from the mm-wave maps were very probably underestimated. Here we presented improved versions of these data. We applied our new code to disk models similar to those derived by Bujarrabal et al. (2005) and compared the predicted profiles with the Herschel spectra (and low- $J$ spectra and maps).

The characteristic temperatures derived by Bujarrabal et al. (2005) were $T\left(R_{\mathrm{kep}}\right) \sim 65 \mathrm{~K}$ (decreasing with the radius as $T \propto$ $\left.1 / r^{0.7}\right)$, where $R_{\text {kep }}$ is the characteristic radius at which the dynamics was found to vary from purely Keplerian to rotation plus a slow expansion (the rotational velocity at $R_{\text {kep }}$ is $\sim 1.65 \mathrm{~km} \mathrm{~s}^{-1}$ ). We found that, as expected, the predicted lines are too low for such temperatures, the $J=16-15$ line intensities being about $1 / 3$ of the observed ones. Assuming $T\left(R_{\mathrm{kep}}\right) \sim 100-150 \mathrm{~K}$ (and keeping the rest of the parameters that describe the structure and physical conditions in the disk practically unchanged), we were able to reproduce the line cores, within a width of about $8 \mathrm{~km} \mathrm{~s}^{-1}$, see Fig. 1. The resulting disk model is summarized in Table 2 (for details on the model and on meaning of the parameters, see Bujarrabal et al. 2005). As we discussed in Sect. 4 and 
Appendix B, the uncertainty in this determination of the typical temperature is moderate, $\sim 20 \%$.

A proper measurement of the temperature in the rotating disk of the Red Rectangle is of particular importance because the physical conditions in these components, crucial for studying the post-AGB ejections and evolution (Sect. 1), are still largely unknown and the physical processes active in them, in particular the thermodynamics, are poorly understood. Indeed, the Red Rectangle disk is practically the only one that has been studied to date. A good estimate of the gas thermodynamics is also fundamental for understanding the complex dynamics of post-AGB disks. A slow expansion was found in the outer parts of the Red Rectangle disk from CO $J=2-1$ maps (Bujarrabal et al. 2005), with velocities of about $1 \mathrm{~km} \mathrm{~s}^{-1}$, comparable to the rotational velocities and much lower than those typical of expanding $\mathrm{PNe}$ and PPNe. For temperatures $\gtrsim 100 \mathrm{~K}$, the thermal velocity dispersion becomes $\gtrsim 1 \mathrm{~km} \mathrm{~s}^{-1}$; when the microscopic velocity is comparable to the rotation velocity (and then to the escape velocity), evaporation becomes able to affect the disk dynamics and introduces a noticeable expansion (e.g. Gesicki et al. 2010; Hollenbach et al. 2000) and could be the mechanism that drives the expansion observed in the disk. Finally, a good knowledge of the properties of the Red Rectangle disk is also important to extend these studies to other similar objects, since $\mathrm{CO}$ mm-wave (still unpublished) observations are revealing a surprisingly high number of extended rotating disks in certain types of post-AGB objects. The interpretation of these data for sources for which we have significantly less information than for the Red Rectangle requires a good knowledge of the properties of the disk in this prototype. Estimates of the disk mass and angular momentum from single-dish mm-wave lines, for instance, cannot be performed for these nebulae if we have only a poor idea of the characteristic temperature.

Even with these new temperature values, the comparison of our synthetic profiles with those observed in the Red Rectangle is not fully satisfactory. The line wing emission, coming from relatively fast gas, is underestimated in intermediate-excitation lines, notably in the emission of the $J=6-5$ transition at LSR velocities of about $\pm 6 \mathrm{~km} \mathrm{~s}^{-1}$. We showed (Sect. 4.1) that this excess cannot be explained assuming the Keplerian or Keplerian-like dynamics expected in the disk, since then the emitting region must be very small and dense, yielding also a very strong intensity in the $J=16-15$ transition (we would expect $T_{\mathrm{mb}} \sim 350 \mathrm{mK}$ at $\pm 7 \mathrm{~km} \mathrm{~s}^{-1}$ ), which is in contrast to the relatively weak wings of this line. This peculiar behavior of the line-wing emission could be explained by postulating a relatively extended and diffuse gas component (typical radius $r \sim 10^{16} \mathrm{~cm}$, equivalent to $\sim 1^{\prime \prime}$, and density $n \sim 10^{5} \mathrm{~cm}^{-3}$ ) that shows moderate typical velocities (between 7 and $15 \mathrm{~km} \mathrm{~s}^{-1}$, depending on the projection angle) probably due to expansion. This component, not to be confused with the slow disk expansion mentioned above, would be associated to the optical nebula and be similar to the expanding structure found in the similar object 89 Her (Sect. 1, Bujarrabal et al. 2007). We discussed (Sect. 4.1) that its mm-wave emission is probably too weak to be detected in our previous mm-wave maps of the Red Rectangle (Bujarrabal et al. 2005), but might be detected in future mapping with higher sensitivity.

Acknowledgements. We are grateful to Mario Tafalla for his help in understanding and checking the Monte Carlo calculations. This work has been supported by the Spanish MICINN, program CONSOLIDER INGENIO 2010, grant "ASTROMOL" (CSD2009-00038).

\section{References}

Alcolea, J., Neri, R., \& Bujarrabal, V. 2007, A\&A, 468, L41

Balick, B., \& Frank, A. 2002, ARA\&A, 40, 439

Bernes, C. 1979, A\&A, 73, 67

Bujarrabal, V., Guibert, J., Nguyen-Q-Rieu, \& Omont, A. 1980, A\&A, 84, 311

Bujarrabal, V., Alcolea, J., Neri, R., \& Grewing, M. 1997, A\&A, 320, 540 Bujarrabal, V., Castro-Carrizo, A., Alcolea, J., \& Sánchez Contreras, C. 2001, A\&A, 377, 868

Bujarrabal, V., Castro-Carrizo, A., Alcolea, J., \& Neri, R. 2005, A\&A, 441, 1031 Bujarrabal, V., van Winckel, H., Neri, R., et al. 2007, A\&A, 468, L45 Bujarrabal, V., Alcolea, J., Soria-Ruiz, R., et al. 2012, A\&A, 537, A8 Castor, J. I. 1970, MNRAS, 149, 111

Castro-Carrizo, A., Bujarrabal, V., Sánchez Contreras, C., Alcolea, J., \& Neri, R. 2002, A\&A, 386, 633

Castro-Carrizo, A., Neri, R., Bujarrabal, V., et al. 2012, A\&A, 545, A1

Cohen, M., Van Winckel, H., Bond, H. E., \& Gull, T. R. 2004, AJ, 127, 2362

de Ruyter, S., van Winckel, H., Maas, T., et al. 2006, A\&A, 448, 641

Frank, A., \& Blackman, E. G. 2004, ApJ, 614, 737

Gesicki, K., Zijlstra, A. A., Szyszka, C., et al. 2010, A\&A, 514, A54

Green, S., \& Thaddeus, P. 1976, ApJ, 205, 766

Hogerheijde, M. R., \& van der Tak, F. F. S. 2000, A\&A, 362, 697

Hollenbach, D. J., Yorke, H. W., \& Johnstone, D. 2000, Protostars and Planets IV, 401

Jura, M., \& Kahane, C. 1999, ApJ, 521, 302

Jura, M., Turner, J., \& Balm, S. P. 1997, ApJ, 474, 741

Koning, N., Kwok, S., \& Steffen, W. 2011, ApJ, 740, 27

Lucas, R. 1976, A\&A, 46, 473

Men'shchikov, A. B., Schertl, D., Tuthill, P. G., et al. 2002, A\&A, 393, 867

Roelfsema, P. R., Helmich, F. P., Teyssier, D., et al. 2012, A\&A, 537, A17

Santander-García, M., Bujarrabal, V., \& Alcolea, J. 2012, A\&A, 545, A114

Schöier, F. L., van der Tak, F. F. S., van Dishoeck, E. F., \& Black, J. H. 2005, A\&A, 432, 369

Sobolev, V. V. 1960, Moving Envelopes of Stars (Cambridge: Harvard University Press)

Soker, N. 2001, ApJ, 558, 157

Van Winckel, H. 2003, ARA\&A, 41, 391

van Zadelhoff, G.-J., Dullemond, C. P., van der Tak, F. F. S., et al. 2002, A\&A, 395,373

Velázquez, P. F., Steffen, W., Raga, A. C., et al. 2011, ApJ, 734, 57

Waelkens, C., Van Winckel, H., Waters, L. B. F. M., \& Bakker, E. J. 1996, A\&A, 314, L17

Waters, L. B. F. M., Trams, N., \& Waelkens, C. 1992, A\&A, 262, L37

Wernli, M., Valiron, P., Faure, A., et al. 2006, A\&A 446, 367

Yang, B., Stancil, P. C., Balakrishnan, N., \& Forrey, R. C. 2010, ApJ, 718, 1062 


\section{Appendix A: Code tests and details}

Our code is designed to treat the excitation of rotational CO levels and other simple molecules in a variety of conditions actually present in the analysis of astronomical observations (Sect. 1). We therefore focus our tests on the validity of the calculations in these cases. It is well known that in quite extreme cases, such as very opaque molecular transitions (particularly of $\mathrm{HCO}^{+}$, van Zadelhoff et al. 2002), the line formation is very difficult to treat and predictions can significantly vary when different codes are used. The treatment of these cases is strongly dependent on the numerical treatment of the line profile, the discretization in cells, etc. Even worse, the results dramatically depend on the properties of the discussed object (size of the cloud, variations of the physical conditions across it, etc.), which in actual cases are not well known. In this context, when opacities are so high, the observational parameters do not practically depend on the properties of the inner regions, but just on the (very complex) structure of a very thin cloud "photosphere", a surface layer that can hardly be adequately described. We therefore focus on tests that request difficult convergence and accurate calculations, but avoid extreme, hardly useful cases.

\section{A.1. 1D models}

We first compared the results from our code with previously published calculations of well-tested codes in 1D cases. For this purpose, we used the 1D version of the program. For comparison, we used calculations published by Lucas (1976) and Bernes (1979), who used well-known codes and applied them to $\mathrm{CO}$ emission in spherical collapsing clouds. The Bernes code is a Monte Carlo approach that has been extended to other molecules and a variety of physical conditions and is very widely used today. The code by Lucas is based on a discrete treatment of the frequencies and directions that approximates the derivatives by finite (very small) differences and the integrals by quadrature forms. To compare results from both papers with our calculations, some interpretation of the assumptions and approaches in these papers is necessary.

We first reproduce the calculations by Lucas (1976). We chose as an example the CO $J=2-1$ and $J=1-0$ profiles calculated for his low-density case and outer velocity equal to $1 \mathrm{~km} \mathrm{~s}^{-1}$. The local velocity dispersion $(1-\sigma)$ is equal to $1 \mathrm{~km} \mathrm{~s}^{-1}$. This is the most representative case, since the level populations are not thermalized and the profile shows a clear self-absorption due to the excitation variation across the cloud. In this case the (constant) physical conditions are density $n=10^{3} \mathrm{~cm}^{-3}$, temperature $T=30 \mathrm{~K}$ and $\mathrm{CO}$ relative abundance $X(\mathrm{CO})=10^{-4}$. We suppose that the published profile is given in units of Rayleigh-Jeans-equivalent temperature (otherwise it is difficult to understand that the 2-1 line, mostly thermalized and opaque, shows this low peak temperature) and that the background cosmic continuum was subtracted from the published profile; both choices are common when predictions are to be compared with radioastronomical observations.

We also assumed that the collisional rates used by Lucas (1976) are those published by Green \& Thaddeus (1976; the Lucas paper was published when these calculations were still in press). We took only the downward collisions from Green \& Thaddeus, the excitation rates were calculated following the microreversibility principle (Sect. 2), but we are not sure that the same was done by Lucas. Finally, we calculated the collisions for the gas temperature, $30 \mathrm{~K}$, by interpolating those published for $20 \mathrm{~K}$ and $40 \mathrm{~K}$.

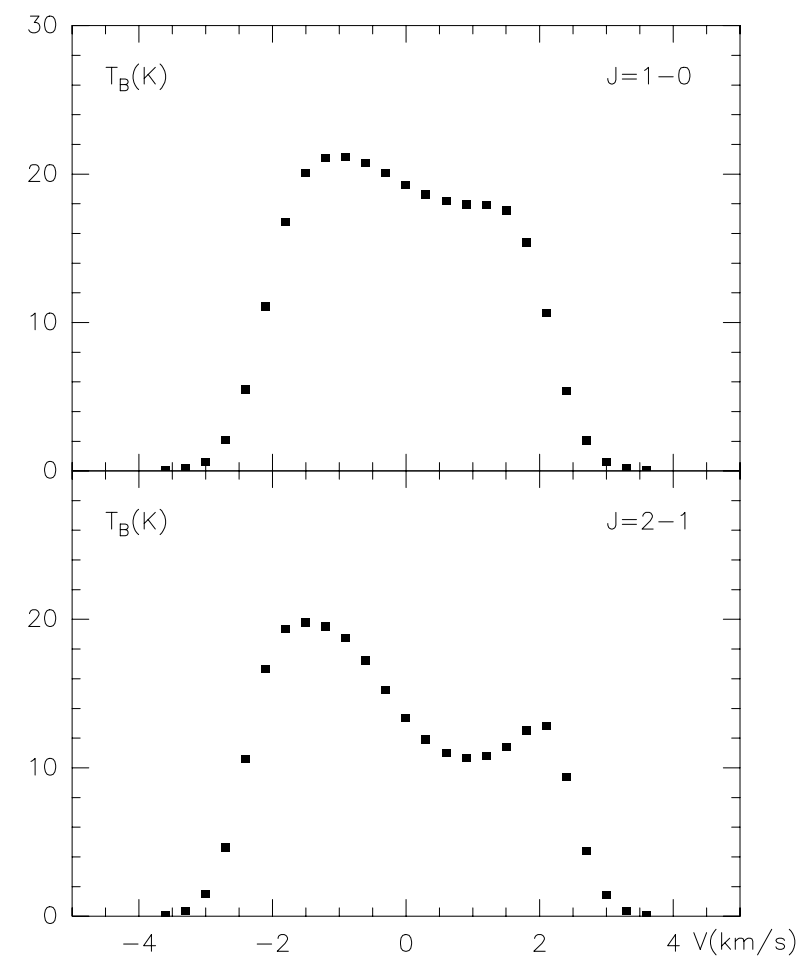

Fig. A.1. Predicted profiles obtained with our code for the ${ }^{12} \mathrm{CO} J=1-0$ and $J=2-1$ lines, for the physical conditions used by Lucas 1976 (the case with density of $10^{3} \mathrm{~cm}^{-3}$ and a maximum radial velocity of $1 \mathrm{~km} \mathrm{~s}^{-1}$ ), to be compared with profiles in Figs. 1 and 2 of Lucas (1976).

Following all these recipes, we resolved the excitation state of the CO levels and calculated the resulting $J=1-0$ and $J=2-1$ profiles, which are shown in Fig. A.1. We checked that convergence is reached in the several parameters used in the code (number of cells, number of considered rays, etc; see Sects. 2.2 and A.2). Clearly, the profiles are identical to those given by Lucas (1976) up to the accuracy that the comparison with the published profiles allows, despite the uncertainties and possible sources of error that remain in the comparison of both codes. We therefore consider the test satisfactory.

The comparison with calculations by Bernes is more complete because this author also published the distribution of the excitation temperature. The model nebula is somewhat larger (with a radius of $3 \times 10^{18} \mathrm{~cm}$ instead of $10^{18} \mathrm{~cm}$ ). Other parameters are $n=2 \times 10^{3} \mathrm{~cm}^{-3}, T=20 \mathrm{~K}$, and $X(\mathrm{CO})=5 \times 10^{-5}$. The velocity field is the same as in Lucas (1976); we assume that the local velocity broadening used by Bernes is also a 1$\sigma$ dispersion. We also applied the microreversibility principle in calculating the collisional rates.

The predicted profile and excitation temperature $\left(T_{\mathrm{ex}}\right)$ for the CO $J=2-1$ transition derived with our code are shown in Figs. A.2 and A.3, to be compared with the corresponding figures in Bernes (1979). We assume that Bernes (1979) gives the profile in units of brightness temperature, in view of the zero-level (equal to about $2.7 \mathrm{~K}$ ) and because the profile peak is equal to the kinetic temperature (as expected in thermalized and opaque lines). Clearly, our results are identical to those obtained by Bernes, within the uncertainties, including the numerical noise that is not negligible in the Monte Carlo approach.

We note that these two examples are very demanding tests for the treatment of radiative transfer and its effects on the molecular level excitation (the key problem), close to the too 
V. Bujarrabal and J. Alcolea: The Red Rectangle rotating disk

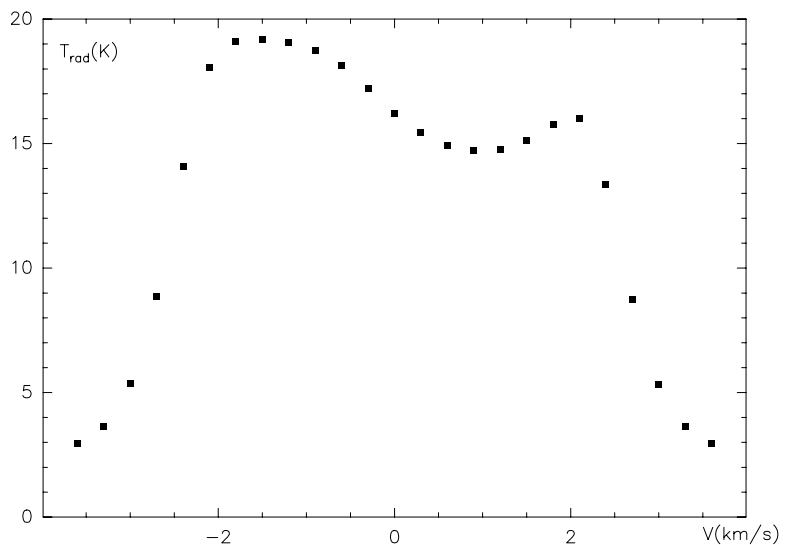

Fig. A.2. Predicted profile obtained with our code for the ${ }^{12} \mathrm{CO} J=2-1$ line, for the physical conditions used by Bernes (1979), to be compared with the corresponding profile in his Fig. 3.

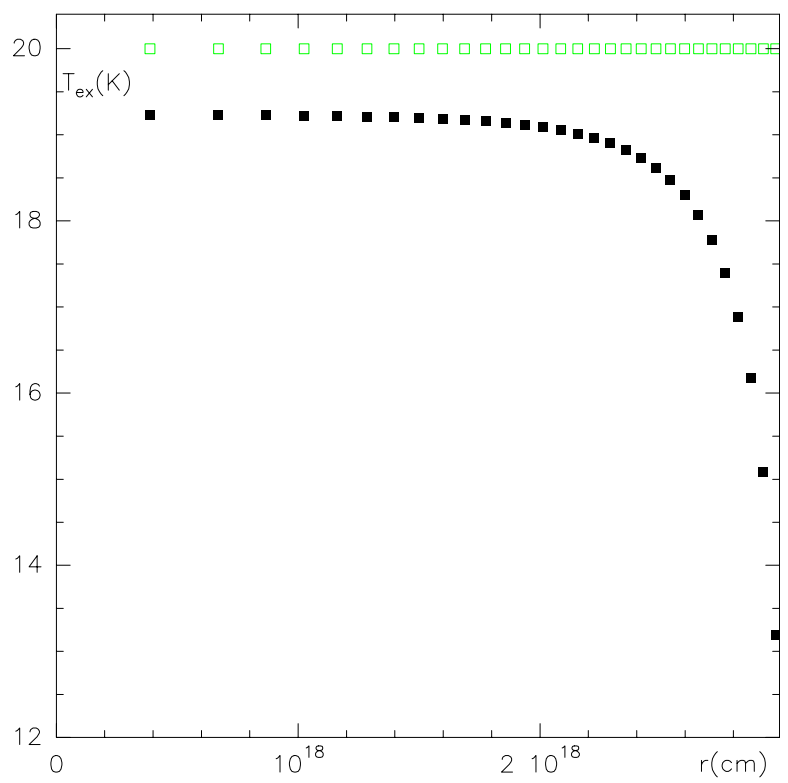

Fig. A.3. Predicted excitation temperature obtained with our code for the ${ }^{12} \mathrm{CO} J=2-1$ line for the physical conditions used by Bernes (1979), to be compared with his Fig. 2. The green, open squares represent the kinetic temperature of the cells.

complex and scarcely practical problems discussed before. The opacities are high, of about 50, and therefore the interaction between different points in the nebula is very complex and the iterative process becomes slow. The differences found between our calculations and those of the previous codes are $\sim 1 \%$ (often indistinguishable from the figures); the largest difference, $\sim 3 \%$, was found in the comparison with the excitation temperature of $J=1-0$ predicted by Bernes (1979) for the outermost boundary of his model cloud (outer $10^{17} \mathrm{~cm}$, where complex population cascades lead to some increase of the 1-0 excitation). We verified that differences found in various runs of our code with different values of the numerical parameters are smaller than $1 \%$; we show in Sect. A. 2 that in some cases our code may show a numerical noise of about $1 \%$ in excitation temperature, which could also appear in the calculations shown here, although it does not obviously manifest itself when different runs are compared. Most probably the main differences with respect to previous works are due to our interpretation of the previous calculations.

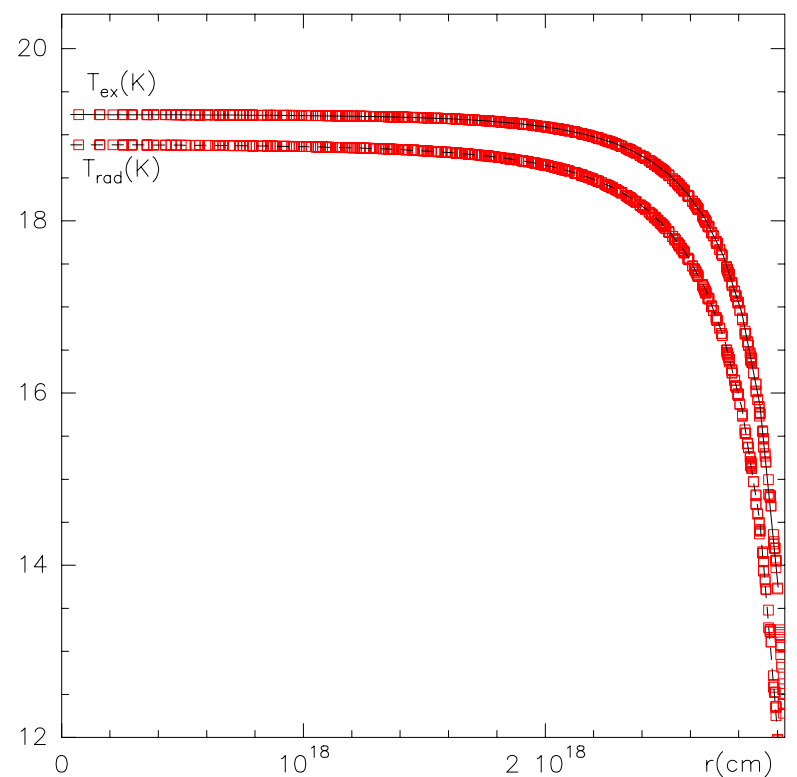

Fig. A.4. Comparison between the excitation temperature $\left(T_{\mathrm{ex}}\right)$ and the equivalent radiation temperature $\left(T_{\text {rad }}\right)$ of the $J=2-1$ transition calculated with the $1 \mathrm{D}$ version of the code (continuous and dashed lines) and with the 2D version (red, empty squares). Calculations performed for the same model as for Fig. A.3 (from Bernes 1979).

Differences of a few percent have often been found when very different codes were compared, see Bernes (1979) and van Zadelhoff et al. (2002). Their origin, except for the numerical noise in Monte Carlo calculations, is difficult to know, as concluded by the quoted authors.

The fact that the tests of our 1D code are positive convincingly shows that it treats the problem correctly.

\section{A.2. Comparison between $1 D$ and $2 D$ codes}

We have mentioned (Sect. 2) that our code easily evolves to a 2D treatment, since, in some way, the two dimensions are included in the code structure. We therefore built a 2D code in which axial symmetry is assumed and the cells are defined in terms of distance to the axis of symmetry and to the equator. We assumed symmetry with respect to the equator in the examples we show here, but it is not necessary in the code (when this symmetry is assumed only one half the cells are needed).

The tests of the 2D code were performed running the $2 \mathrm{D}$ code with the same conditions as assumed in the $1 \mathrm{D}$ examples. In other words, the cell definition and radiative transfer treatment were performed fully in 2 dimensions, with their coordinates given by the distances to the axis and equator, but the physical conditions in the cloud satisfy spherical symmetry. In this way, the 2D treatment can be checked by comparing it with 1D code results, whose accuracy was discussed above (Sect. A.1). A few non-local 2D calculations of CO excitation have been published for cases similar to those we are interested in, e.g. Hogerheijde \& van der Tak (2000), but the lack of details on the calculation and nebula model prevents a careful comparison. These authors also checked their code by comparing its CO predictions with the 1D results from Bernes (1979).

In Fig. A.4 we compare the predictions of the excitation temperature of the CO $J=2-1$ transition derived from our 1D code (continuous black line) and those from the 2D code (open red squares). We used the same nebula model as in Sect. A.1 to compare our results with those by Bernes (1979), including the 
collisional rates. We considered a total of 40 cells in the $1 \mathrm{D}$ calculations and 900 cells in the 2D case. We also show in this figure the equivalent temperature of the radiation field $\left(T_{\mathrm{rad}}\right)$ seen in each cell (lower discontinuous, black line and red squares); of course, the excitation temperature is placed between this radiation field temperature and the kinetic one $(20 \mathrm{~K})$, its value is given by the collisional/radiative de-excitation probability ratio.

The comparison is again very positive. We can see some numerical errors (differences in excitation temperatures for cells at the same distance to the center, between different cells of the $2 \mathrm{D}$ code and with respect to the $1 \mathrm{D}$ calculations), but they are very moderate, $\lesssim 1 \%$. The errors in $T_{\mathrm{ex}}$ are comparable to those found for $T_{\text {rad }}$, and calculating the radiation intensity in just one point per cell is probably the source of the whole uncertainty.

We also performed calculations for conditions under which the errors may be slightly larger. When the macroscopic velocity field becomes higher, the velocity variations within a given cell can be relatively large. In such cases, errors may appear in the calculation of the relative velocities of two cells, resulting in errors in the calculations of the emission and absorption rates at the relevant frequencies.

In Fig. A.5 we show the excitation and radiation temperatures for the same case as before but increasing the maximum velocity to $4 \mathrm{~km} \mathrm{~s}^{-1}$. For calculations in the upper panel, we took 40 and 100 cells for the $1 \mathrm{D}$ and the 2D models respectively (symbols are the same as in Fig. A.4). As we see, differences slightly higher than $1 \%$ are noticeable in the $2 \mathrm{D}$ calculations and with respect to the $1 \mathrm{D}$ results. Even some systematic underexcitation of the 1D calculations can be seen in the figure. As mentioned, the reason for the appearance of some numerical noise is that the cells are relatively large (which is particularly the case of the spherical cells). The results converge for higher numbers of cells, as we can see in Fig. A.5, lower panel, for which we used 75 and 900 cells for the $1 \mathrm{D}$ and $2 \mathrm{D}$ cases. In this panel we also show 1D calculations using more recent collisional rates (pointed, green line), taken from the LAMBDA-database ${ }^{2}$ (Wernli et al. 2006). As we see, the results are different from those using calculations by Green \& Thaddeus (1976), as in our previous runs, but not dramatically, by about $5 \%$ in $T_{\mathrm{ex}}$. From now on, we use these more accurate rates in our tests. We finally show still more recent calculations from the same database (Yang et al. 2010), dashpoint line; the differences are still smaller, $\lesssim 2 \%$, showing the moderate changes in the final calculations often found when new collisional rates are incorporated.

For higher values of the macroscopic velocity, convergence becomes increasingly difficult. As we show below, in these cases calculating the level populations with the well-known LVG approximation is much more efficient.

\section{A.3. Comparison with LVG calculations}

The well-known LVG approximation holds when in a cloud the module of the macroscopic velocity increases monotonically with radius, with a gradient steep enough to ensure that points decoupled radiatively are still close and expected to show similar physical and excitation conditions. As mentioned in Sect. 2, the averaged radiation intensity in any point, which rules the radiatively stimulated rates in the transitions, is then a local parameter and the treatment becomes equivalent to the definition of an escape probability of photons, which represents the percentage of photons emitted by the gas that actually escapes from the cloud in spite of self-absorption (trapping). LVG codes are therefore

\footnotetext{
2 http://www.strw.leidenuniv.nl/ moldata
}

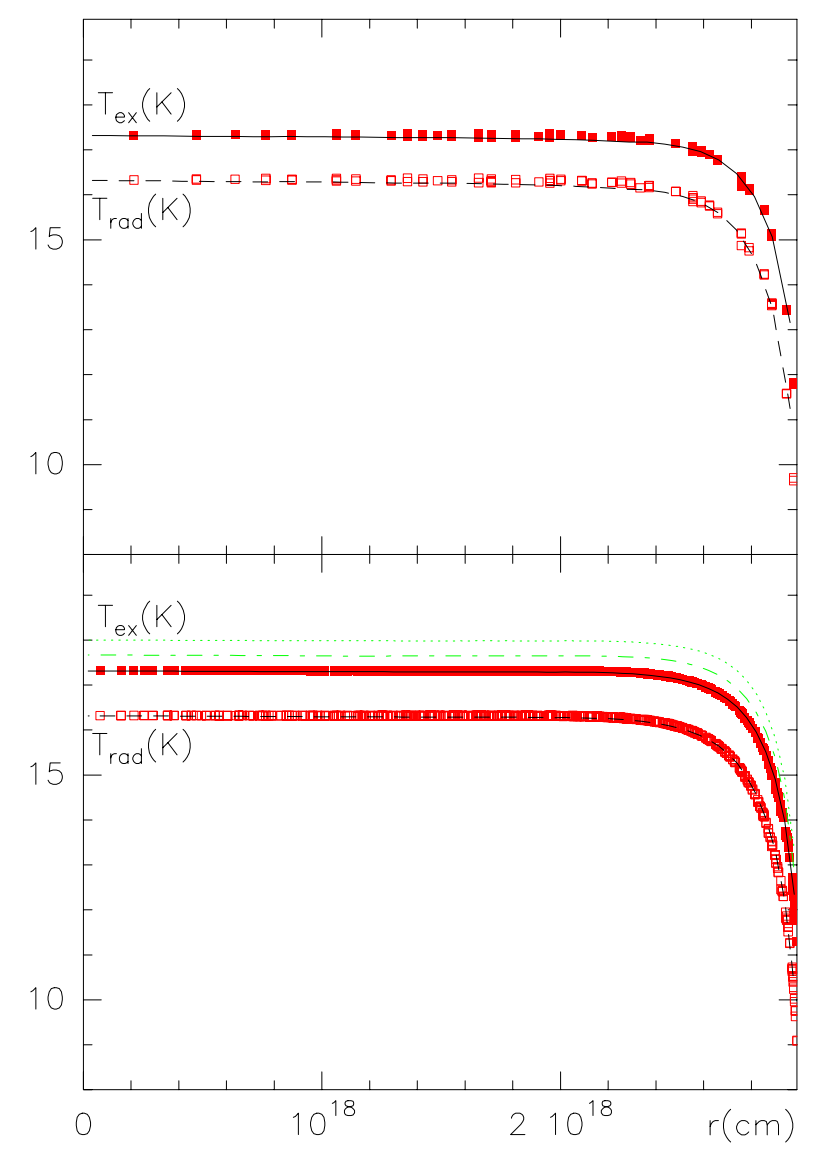

Fig. A.5. Calculations similar to those performed for Fig. A.4, but for a model cloud in which the highest inward velocity is equal to $4 \mathrm{~km} \mathrm{~s}^{-1}$. Upper panel: using 40 and 100 cells for the 1D and 2D calculations. Lower panel: using 75 and 900 cells; the green point and dash-point lines represent $1 \mathrm{D}$ calculations when more recent collisional rates are used (see text). Note the improvement in the calculation noise when more cells are used and the moderate influence of collisional rates.

very fast and relatively simple, though they incorporate the main ingredients of the problem. It is also known that this approximation yields reasonable values of the level population even when the conditions are only marginally satisfied (although the resulting line profile must in any case be calculated by solving the exact radiative transfer equation for the derived level populations). Because of the inherent radiative decoupling introduced by the LVG approximation, it can easily be extended to 2- or even 3D calculations, the (local) excitation being in fact independent of the geometry of the cloud at large scale.

We performed LVG calculations for the nebula models we studied in the previous subsections and other similar ones. In the LVG approximation, the effects of the nebula size and macroscopic velocity field enter the calculation of the opacity via the factor $r / V, \tau \propto r / V$, where $r$ and $V$ are the distance to the center and the expansion or collapse velocity at this distance; the local velocity dispersion is neglected. Since we will apply this treatment to cases in which the local velocity is not negligible compared to the macroscopic one, we substituted in our calculations the value of $V$ by $V+\Delta V / 2$, where $\Delta V$ is the width at half-maximum of the local velocity dispersion.

In a first case, we considered the same nebula model as Bernes (1979), see Sect. A.2. We recall that this time we did not use the collisional rates by Green $\&$ Thaddeus but the more recent calculations (Sect. A.3). In Fig. A.6, upper panel, we compare results from our non-local 'exact' treatment (black squares) 


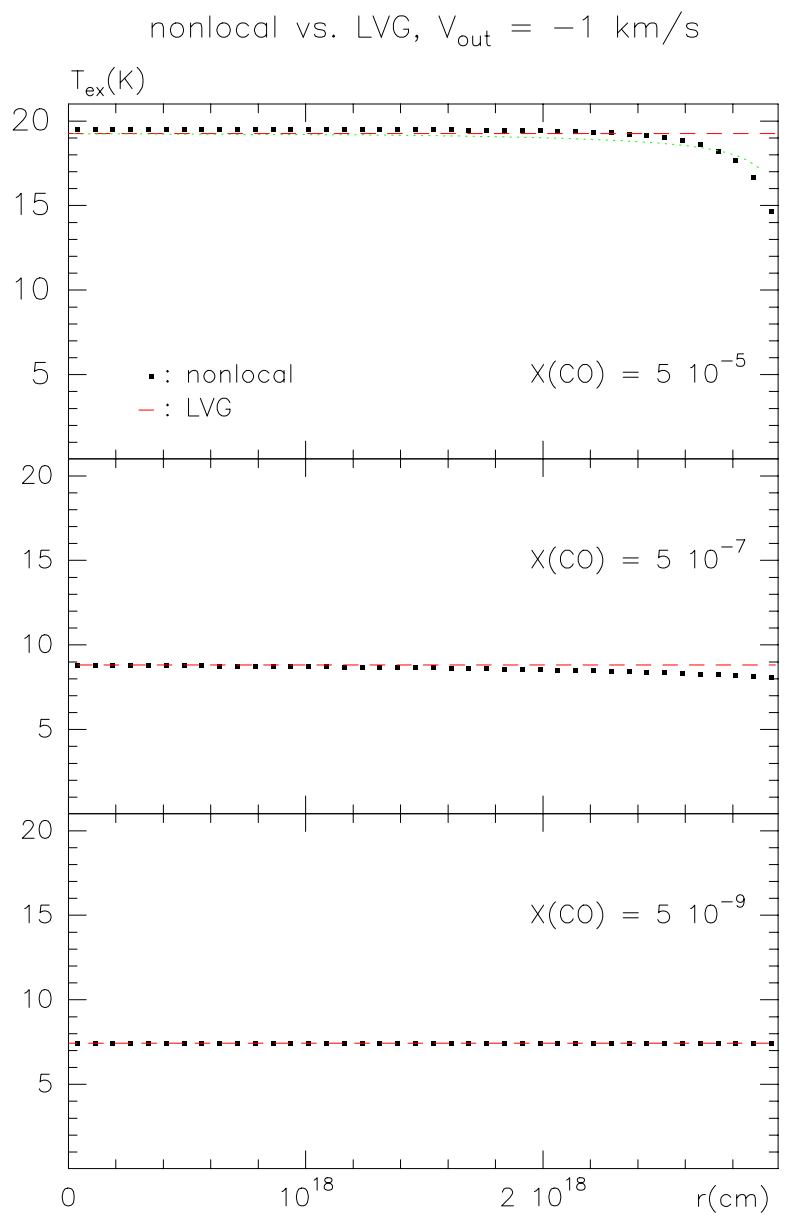

Fig. A.6. Comparison between calculations performed using our nonlocal treatment of radiative transfer (squares) and the LVG approximation for a cloud model similar to that used previously (from Bernes 1979) for different values of the relative $\mathrm{CO}$ abundance (which led to an optically thin case for $X(\mathrm{CO})=5 \times 10^{-9}$, lower panel). The green dotted lines represent a modification of the LVG approach trying to simulate boundary effects.

with those from the LVG approximation (red dashed line). The LVG results are surprisingly similar to those from the non-local code, even if the highest macroscopic velocity is just comparable to the local dispersion. In reality, the LVG predictions are independent of the point of the nebula we are considering, because in this nebula model the value of $r / V$ is constant. Therefore, in the outer regions, where very little photon trapping occurs because we are very close to the nebula boundary, the non-local code gives a somewhat lower excitation (approaching the values obtained for lower abundances, see below) and the LVG limit of very low local velocity dispersion cannot deal with this effect. It is possible to define "equivalent" values of $r$ to simulate this effect in the LVG code and improve the predictions in outer regions. We do not discuss these procedures here, but just show (pointed green lines) calculations in which we took for the outer points an equivalent radius of the cloud equal to the distance between the point and the outer circumference in the direction perpendicular to the radius direction between the point and the cloud center; we point out that the results are somewhat improved in the outer regions but not yet fully satisfactory.

In the other panels of the figure, we show the same calculations for lower values of the $\mathrm{CO}$ relative abundance, and therefore of the opacities. As expected, the excitation temperature

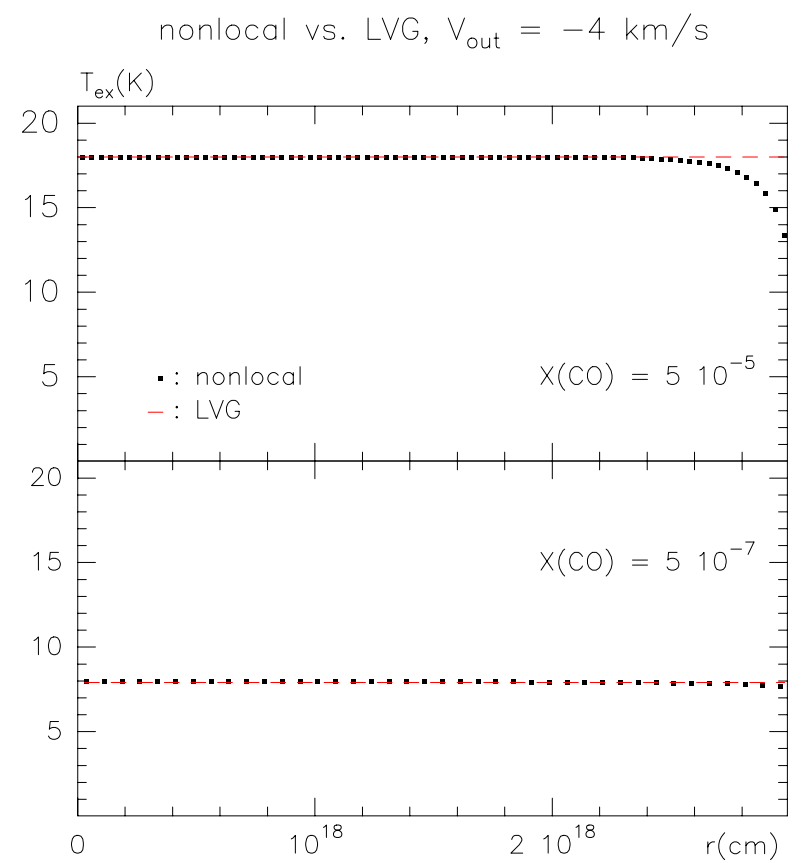

Fig. A.7. Comparison between calculations performed using our nonlocal treatment of radiative transfer (squares) and the LVG approximation, similar to those of Fig. A.6, except for a higher value of the outer collapse velocity, in this case equal to $-4 \mathrm{~km} \mathrm{~s}^{-1}$. The very low opacity case is identical to that of Fig. A.6 (lowest panel) and is not displayed again.

decreases (because of the less frequent trapping) and the agreement with LVG improves (because in the optically thin limit the statistical equilibrium equations, which give the level populations, become independent of the radiative transfer treatment).

We have seen that when the local and macroscopic velocities are practically the same, far from the LVG assumptions of negligible local $\Delta V$, the predictions of the simple LVG codes are very reasonable. Only the decreasing excitation toward the edge of the cloud in very opaque cases can hardly be accounted for by an LVG treatment, and therefore the asymmetries of the profiles seen in Sect. A.1 cannot be obtained from it.

We performed calculations in other cases. In Figs. A.7 and A.8, we can see the same model but assuming a higher outer collapse velocity $V_{\text {out }}$ of -4 and $-8 \mathrm{~km} \mathrm{~s}^{-1}$. As expected, the LVG predictions are still better. Again, for lower opacities the coincidence is absolute.

We also repeated the calculations for the case where the macroscopic velocity is equal to zero, Fig. A.9, and the LVG approach is forced to maximum. (As said before, in this case the local velocity dispersion in fact substitutes the macroscopic velocity in our LVG calculations.) For high optical depths (high abundance) the agreement is good, because the line is practically thermalized (and, of course, the agreement is very good for very low opacities). But for cases with moderate opacities, where the radiative transfer effects are relevant, there is a significant difference between the non-local and LVG codes; in any case, the difference is not extreme, only of about $20 \%$ in terms of $T_{\text {ex }}(2-1)$, a value that could be improved if we changed the definition of the LVG-equivalent velocity.

We also performed a calculation similar to our standard case (Bernes nebula model), but with a slowly increasing value of the radial velocity (Fig. A.10). This is particularly interesting for studying AGB circumstellar envelopes, which present a velocity law of this kind. We considered a law $V(r)=V_{\text {out }} \times\left(r / R_{\text {out }}\right)^{\epsilon}$. A 


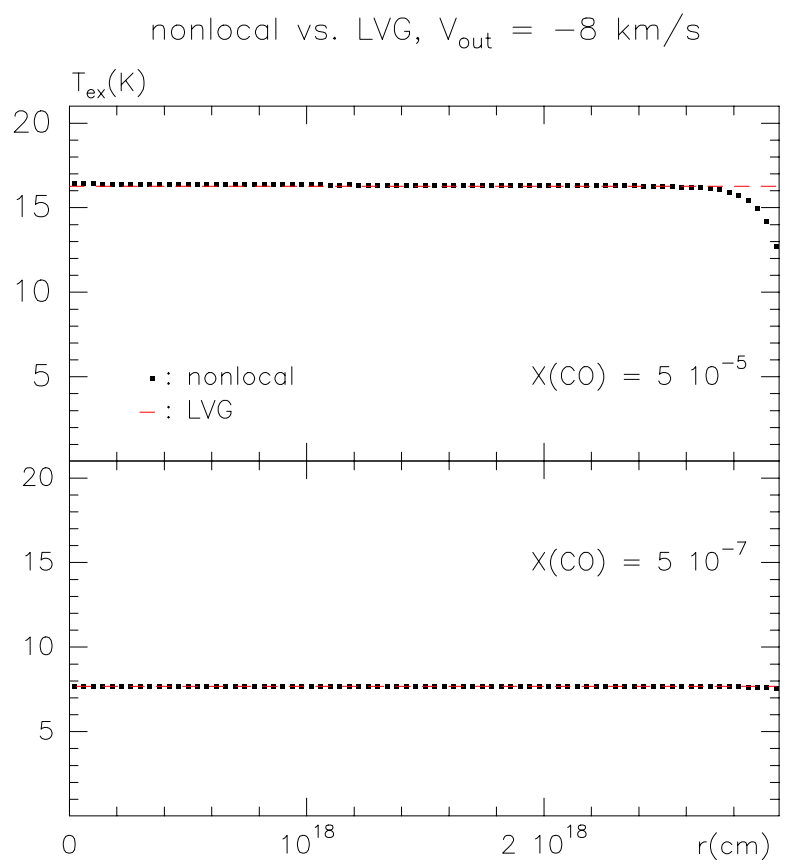

Fig. A.8. Same as Fig. A.7 but for an outer collapse velocity of $-8 \mathrm{~km} \mathrm{~s}^{-1}$.

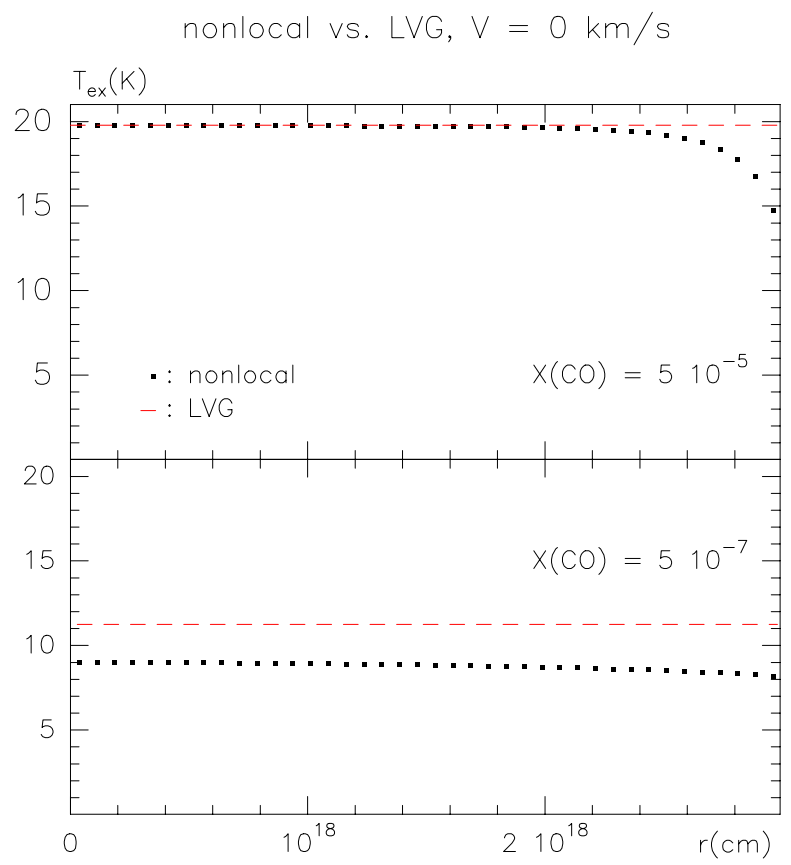

Fig. A.9. Same as Fig. A.7 but without the macroscopic velocity field.

constant logarithmic velocity gradient was chosen because this is the parameter that actually enters the calculation of the LVG escape probability. We took a quite extreme case with $\epsilon=0.1$. In a law of this kind, the variation of the velocity along the radial direction is slow and all spherical layers interact radiatively in that direction (not in the tangential one). Results in Fig. A.10 show a good behavior of the LVG approach, except for the very outer regions. Here $r / V$ is not constant across the nebula.

Finally, we performed calculations for higher- $J$ transitions with our non-local code and compered them with LVG results. We show here as an example calculations of the $J=6-5$ excitation for our standard cloud model (from Bernes 1979), but for variable $\mathrm{CO}$ abundances and with ten rotational levels. (We

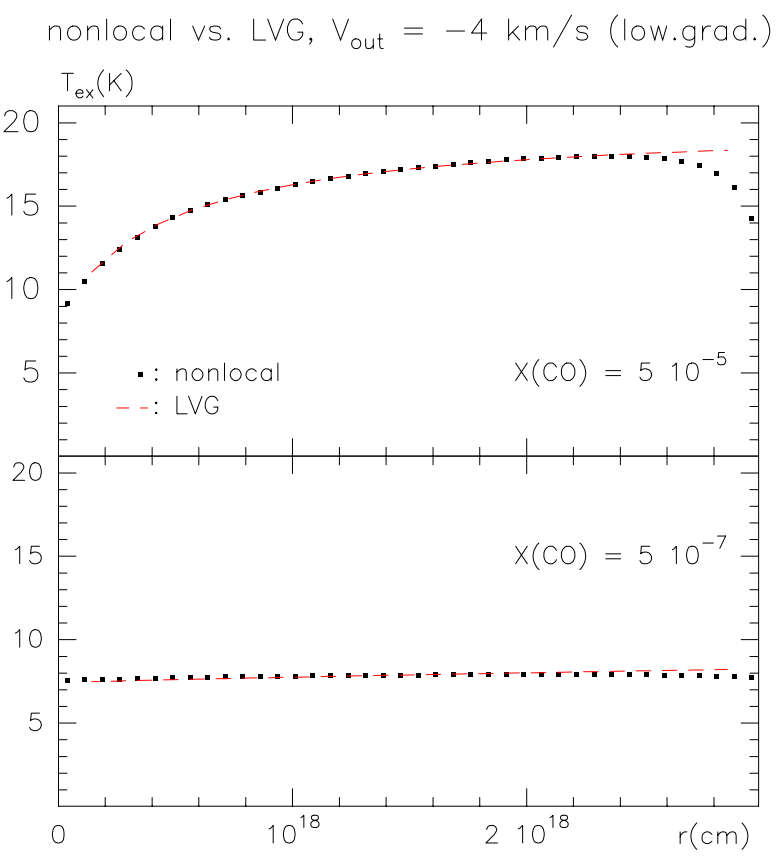

Fig. A.10. Same as Fig. 7 but assuming a slowly increasing macroscopic velocity, defined by a constant logarithmic gradient of the velocity of 0.1 .

verified that the results for $J=2-1$ in these calculations are almost identical to those previously reported with only six rotational levels.) We show the results in Fig. A.11. Except for the edge effects in the very optically thick case, the LVG results are identical to those from the non-local treatment. The excitation temperature increases close to the cloud edge in the non-local calculations for high abundance, which is because transitions below $J=6-5$ are very opaque and those above it are optically thin and, therefore, photon cascades tend to relatively overpopulate levels with $J \sim 6$ in the outer layers. This test is very demanding, because the opacity is very high for $J=5-4$ and lower transitions but the excitation state is low for high- $J$ levels (the energy of the $J=6$ level is $116 \mathrm{~K}$ but the kinetic temperature in the model is just $20 \mathrm{~K}$ ). The comparable excitation given by the non-local and LVG calculations confirms the accuracy of our calculations also for high- $J$ levels and even for strong underexcitation. Similar tests are difficult to perform by comparison with previous works because of the lack calculations for these high transitions.

In summary, we have seen that the LVG approximation is quite accurate in producing the excitation conditions of molecular gas for clouds showing radial velocities, even if they are very low and the conditions of the approximations are in fact not satisfied. The good agreement of our calculations with the LVG ones in all reasonable cases must also be considered as an independent test of our non-local code.

\section{A.4. Axisymmetric nebulae}

We applied our 2D code to clouds with axial, but without spherical symmetry. In all cases discussed here, we assumed symmetry with respect to the equatorial plane. We considered as basic example the same physical conditions as in previous subsections, i.e., the Bernes (1979) nebula model with constant density, temperature, and $\mathrm{CO}$ abundance $\left(n=2 \times 10^{3} \mathrm{~cm}^{-3}, T=20 \mathrm{~K}\right.$, $\left.X(\mathrm{CO})=5 \times 10^{-5}\right)$, and radial velocity increasing linearly up to a value of $1 \mathrm{~km} \mathrm{~s}^{-1}$ in the nebula limit. One of the radii (equatorial 


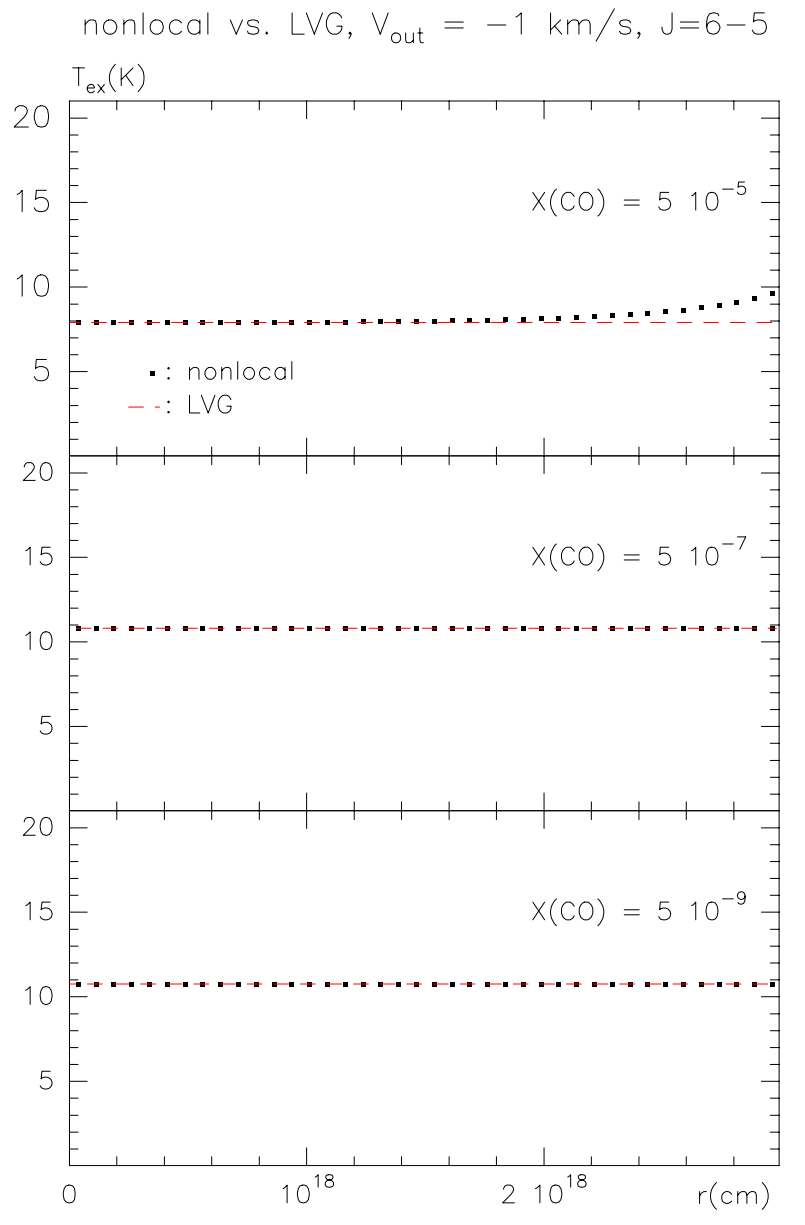

Fig. A.11. Same as Fig. A. 6 but for transition $J=6-5$ and including a total of ten rotational levels.

or axial) is kept equal to $3 \times 10^{18} \mathrm{~cm}$ and the other is decreased to $10^{18} \mathrm{~cm}$. The result is a strongly prolate or oblate structure, in which the velocity gradients are different in the equatorial and axial directions (while the final velocities are the same).

We show the resulting $T_{\text {ex }}(2-1)$ in Figs. A.12 and A.13 (red squares), continuous red lines join points along the equator and along the axis. For comparison we show the results from the spherical cases with cloud radii of $10^{18}$ and $3 \times 10^{18} \mathrm{~cm}$ (continuous black lines); as expected, the prolate and oblate structures show intermediate excitations between these two extreme cases. The points with the lowest values of $T_{\mathrm{ex}}$ are always close to the edge.

Finally, we incorporated Keplerian rotation in our code (which is our goal in order to apply it to rotating disks). We considered rotation around the symmetry axis in our oblate structure (as defined above) and the rotation velocity was assumed to vary inversely with the square-root of the distance to the axis, with a velocity of $0.3 \mathrm{~km} \mathrm{~s}^{-1}$ in the outer regions, and reaching values higher than $4 \mathrm{~km} \mathrm{~s}^{-1}$ in the innermost code cells. (This field would correspond to rotation around a central compact mass of a few solar masses, but does not really represent a stable Keplerian movement, because the total mass of the cloud is already larger than this value.) The results can be seen in Fig. A.14; again we represent the $J=2-1$ excitation temperatures derived for the rotating cloud and for our standard spherical clouds (continuous black lines), as in Figs. A.11 and A.12.

We recall that to facilitate the comparison with previous figures, we have assumed constant density. That is usually not the

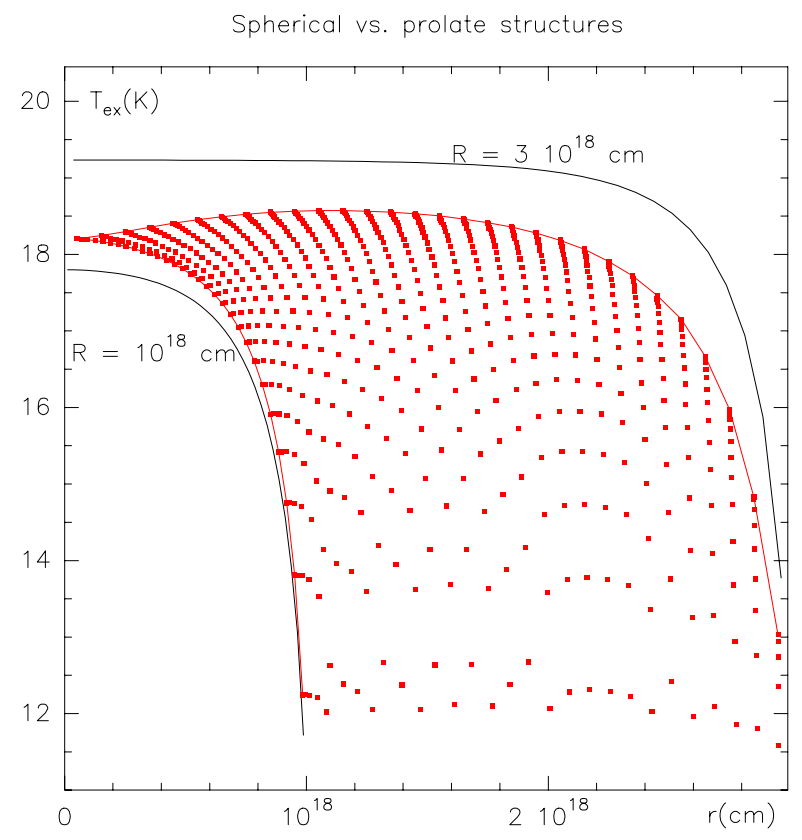

Fig. A.12. Red squares: 2D calculations of the $J=2-1$ excitation temperature performed for a model cloud similar to that discussed previously (the Bernes model cloud) but showing a strongly prolate structure. The red lines join points along the axis (those reaching higher values of the distance to the center, $r$ ) and along the equator. Continuous black lines: calculations for spherical clouds with radii equal to the maximum and minimum radius of the prolate cloud.

case in actual rotating disks; when we assume the density to increase toward the center (with $1 / r$ or $1 / r^{2}$ ), the excitation is high in the central regions and the $J=2-1$ line thermalizes in them.

We also represent in Fig. A.14 predictions from our LVG code (dashed red line). For these calculations, we took the absolute values of the distance to the center and of the tangential velocity to calculate the factor $r / V$ (as discussed in Sects. A.3, including the correction to the velocity due to the local dispersion) and $\epsilon=1$. Evidently, the LVG results give a rough idea of the expected excitation temperatures, but errors can be very large in this case (larger than 30\%), even if we just compare the LVG results with the non-local predictions for points along the equator (squares joined by the upper red line). The reason is that in rotating clouds radiative interaction between very distant points can take place, in a complex long-distance interaction pattern, and the LVG approximation is not valid at all. Particularly in inner points, where rotation is fast, the LVG procedure implies that radiative interaction only takes place within a very small region, though in reality even line radiation from the edges of the cloud can be absorbed in these innermost layers.

In summary, the LVG calculations have been found to give a good representation of the molecular excitation in spherical and axisymmetric clouds showing radial velocity fields, even in extreme conditions (including the case without a macroscpic velocity field). However, in a rotating cloud the LVG results only give an idea of the true $T_{\text {ex }}$ values, particularly in the inner regions that are in fast rotation. It is difficult to imagine how the standard LVG formalism could be modified to obtain a better approximation to the radiative transfer in this case. 


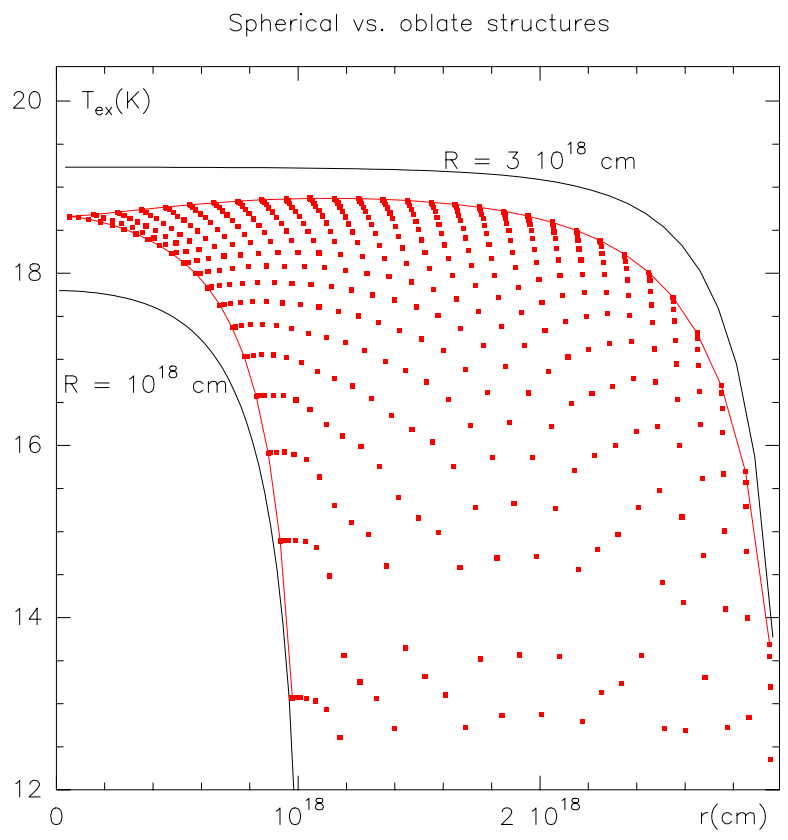

Fig. A.13. Red squares: 2D calculations of the $J=2-1$ excitation temperature performed for a model similar to that of Fig. A.12 but now showing a strongly oblate structure. The red lines join points along the equator (those reaching higher values of the distance to the center, $r$ ) and along the axis. Continuous black lines: calculations for spherical clouds with radii equal to the maximum and minimum radius of the prolate cloud.

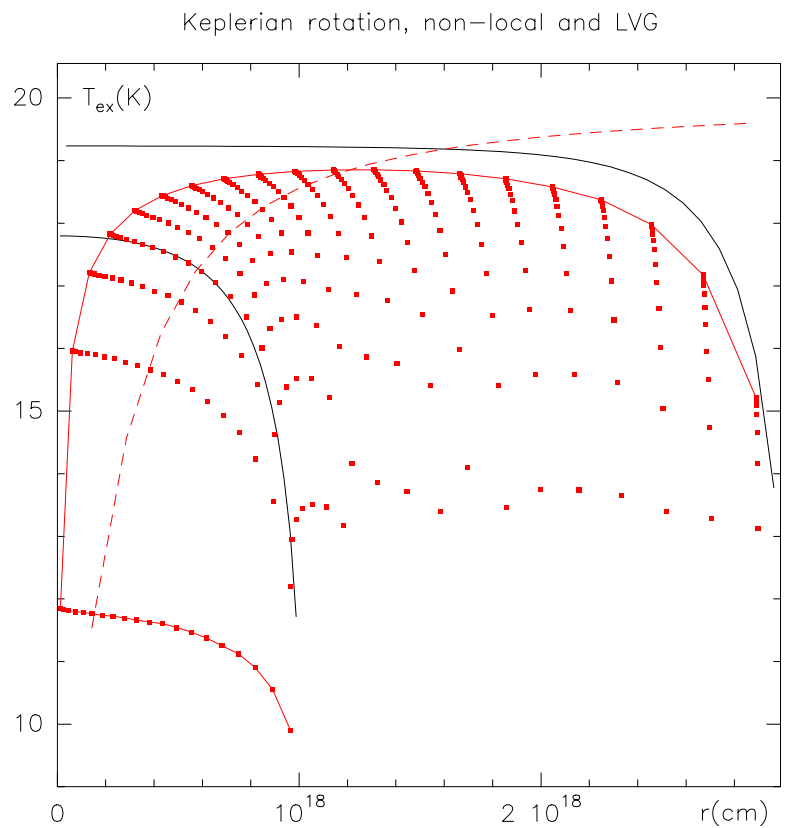

Fig. A.14. Red squares: calculations similar to those shown in Fig. A.13 but assuming that the oblate structure is in Keplerian rotation. The dashed red line represents LVG calculations for the distance and absolute value of the velocity used here, which do not approximate the "exact" code results.

\section{Appendix B: Line predictions for alternative models of the Red Rectangle}

We investigated the variations of the predicted intensity of the lines discussed here as a function of the physical conditions in the disk, notably of the gas temperature, which is the parameter

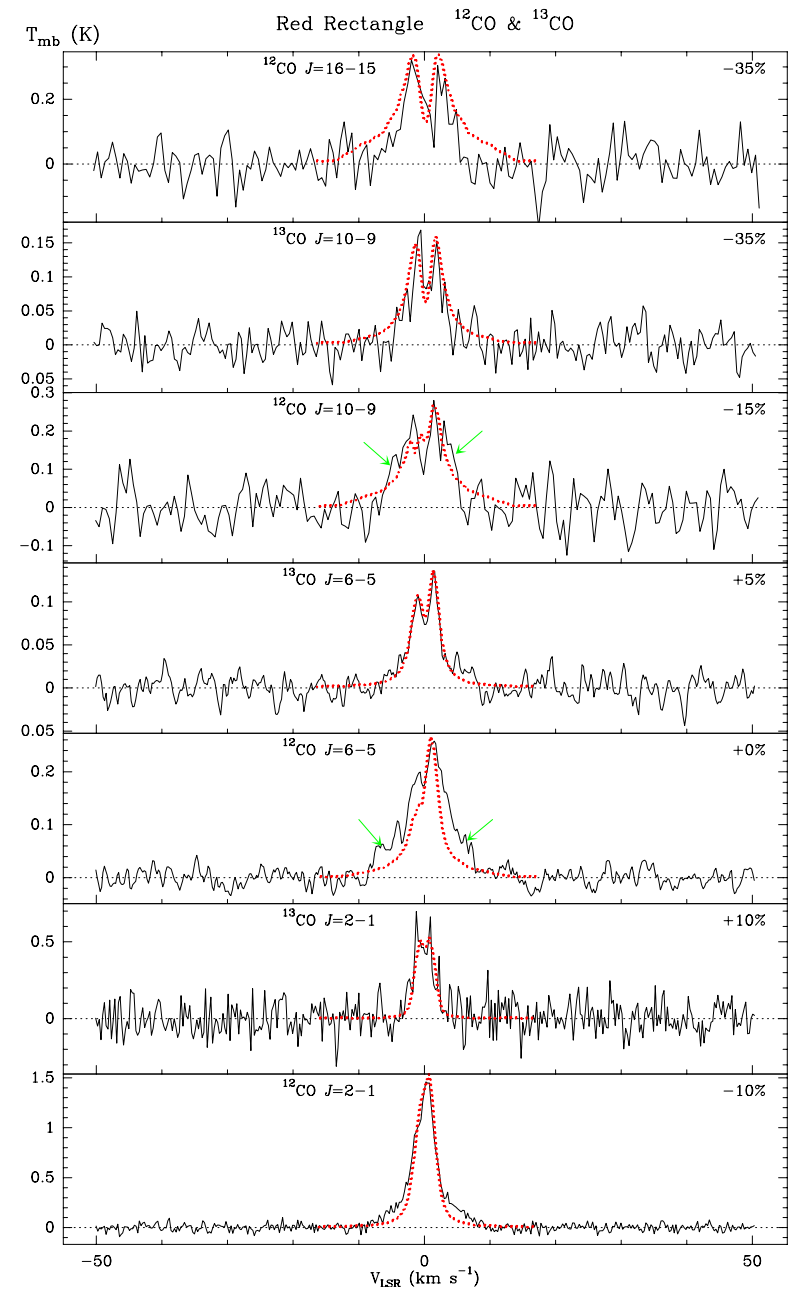

Fig. B.1. Profiles of the observed mm- and submm-wave transitions (black) and the predictions of our code (red points), assuming that the slope of the temperature profiles increases to 1.3 instead of 1 , as in our standard model, Fig. 1, Table 2. The symbols have the same meaning as in Fig. 1; note the values of the multiplicative parameter.

discussed in more detail in this paper. In Sect. 4.1, we have already presented the line intensity variation if we include a central region (at a distance to the axis smaller than $2 \times 10^{15} \mathrm{~cm}$ ) with temperature and density higher than in our standard model by a factor 2. Figure 2 showed that this new component can help to explain the high line-wing emission in intermediate- $J$ transitions, but yields too strong emission in the $J=16-15$ line, therefore the problem of the too high line-wing intensity persists. We present here a similar case, in which the values of $T\left(R_{\mathrm{kep}}\right)$ are the same as in our standard model, but we increase the slope of the dependence of $T$ with the distance to the center from 1 to 1.3. This is equivalent to an increase in the temperature by $20 \%$ at the point $R=R_{\mathrm{kep}} / 2$ and by $50 \%$ at $2 \times 10^{15} \mathrm{~cm}$ from the star. Results are shown in Fig. B.1. Clearly, the predicted intensity for the $J=16-15$ transitions is already too high and this new law leads to a significant increase in the $J=6-5$ line wings. As discussed in Sect. 4.1, models of rotating disks of this kind cannot explain the $J=6-5$ line wings and the $J=16-15$ profile.

We investigated the deviations of the predictions from the observed profiles assuming changes in the temperature from our best-fitting model. In Figs. B.2 and B.3, we show predictions assuming that the temperature varies by $\pm 20 \%$ with respect to our standard model, while the rest of the parameters remain the 


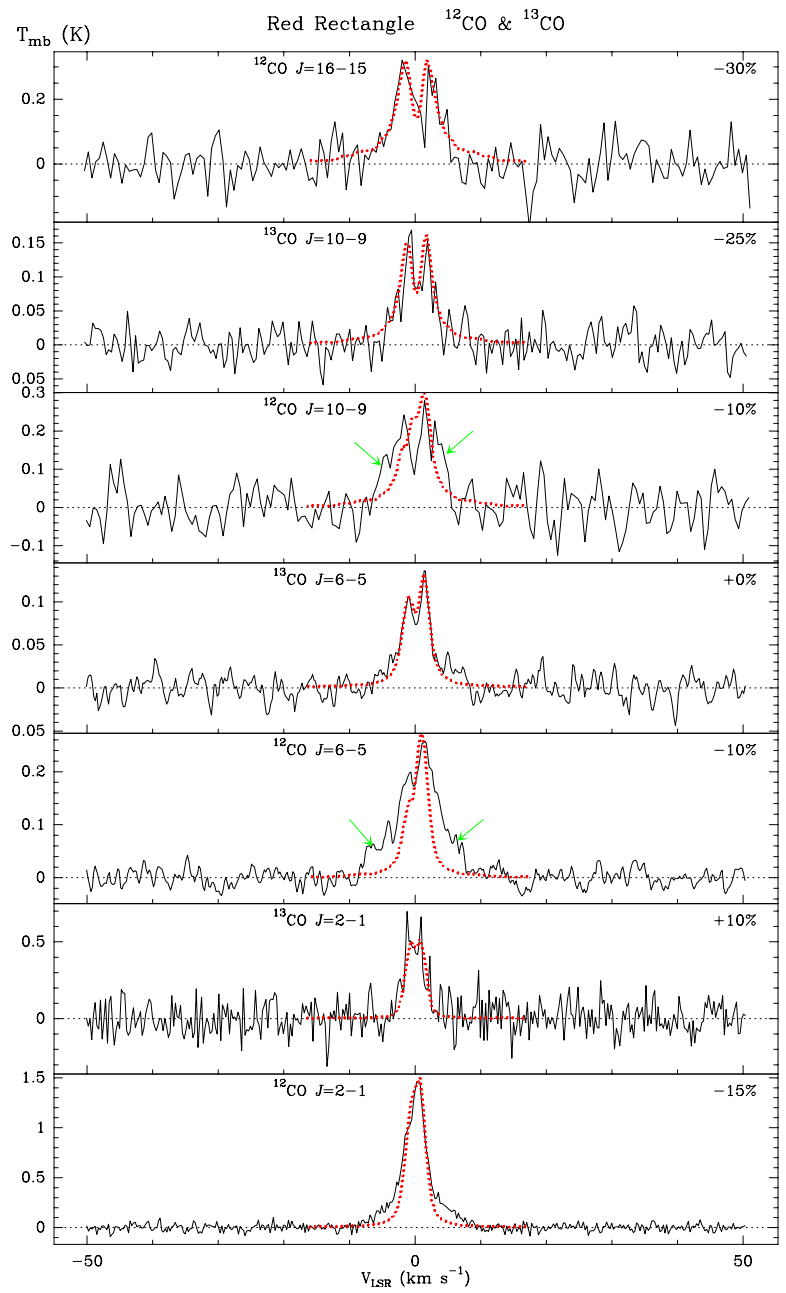

Fig. B.2. Profiles of the observed mm- and submm-wave transitions (black) and the predictions of our code (red points), assuming that the temperature increases by $20 \%$ with respect to our standard model, Fig. 1, Table 2. The symbols have the same meaning as in Fig. 1; note the values of the multiplicative parameter.

same. These variations are sufficient to yield predictions incompatible with the data (though not by a very large factor), taking the calibration uncertainties into account, and can be considered in these conditions as a measure of the uncertainty in the estimated temperature values.

The low- $J$ transitions depend very little on the kinetic temperature for the investigated cases.

Finally, we considered a nebula model in which both the density and temperature vary. We assumed that the density increases by a factor 2 and at the same time that the temperatures decreases

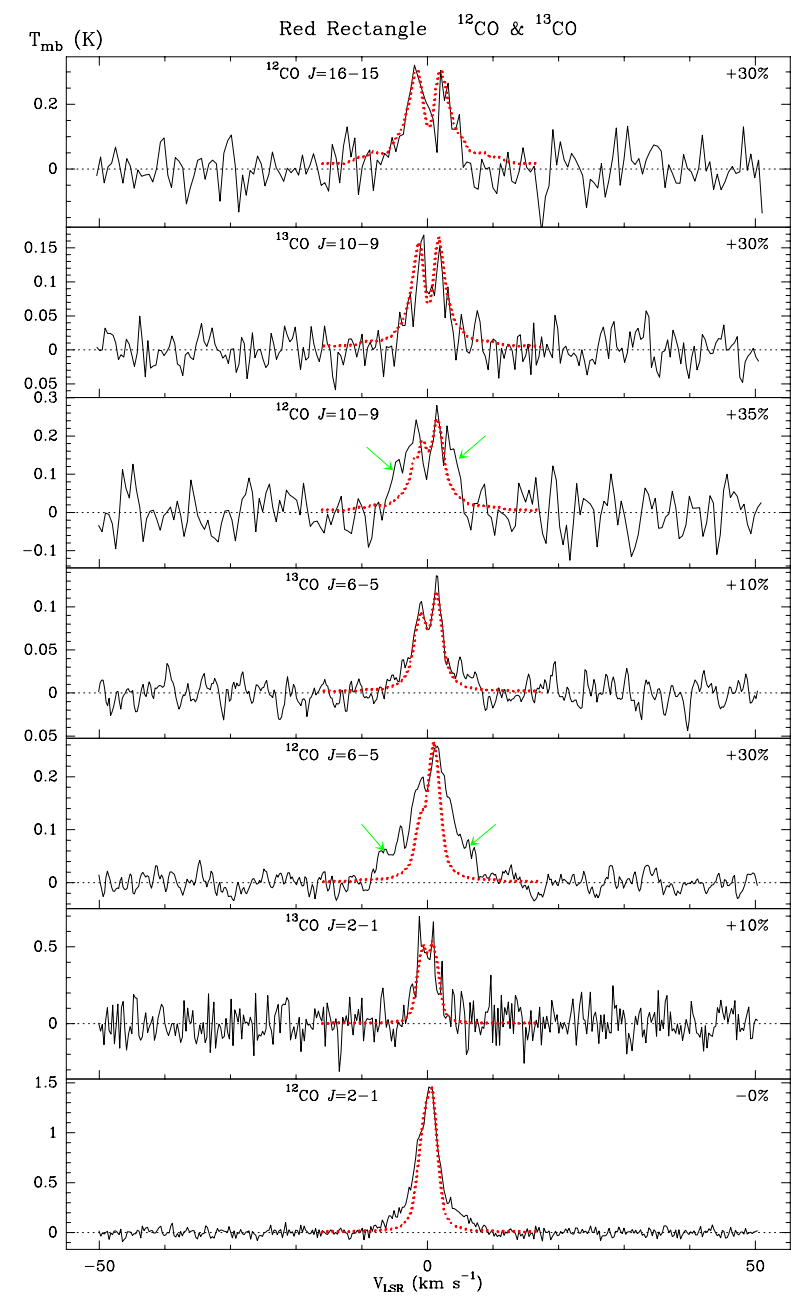

Fig. B.3. Profiles of the observed mm- and submm-wave transitions (black) and the predictions of our code (red points), assuming that the temperature decreases by $20 \%$ with respect to our standard model, Fig. 1, Table 2. The symbols have the same meaning as in Fig. 1; note the values of the multiplicative parameter.

by a $30 \%$. The results are very similar to those shown in Fig. B.3, the comparison with the observations is not acceptable, but now for a variation in the temperature of a $30 \%$. We conclude that allowing variations in the density relaxes the uncertainties in determining the temperature, but not by a large factor, from $20 \%$ to $30 \%$ in this case. Deeper changes in the model would contradict our initial intention of keeping the nebula model deduced from mm-wave maps (Bujarrabal et al. 2005) invariable as far as possible. 